\title{
Cellular and humoral immune responses following SARS-CoV-2 mRNA vaccination in patients with multiple sclerosis on anti-CD20 therapy
}

\author{
Sokratis A. Apostolidis $\mathbb{1}^{1,2,3,14}$, Mihir Kakara4,5,14, Mark M. Painter ${ }^{1,3,6,14}$, Rishi R. Goel ${ }^{13,6,14}$, \\ Divij Mathew ${ }^{1,3,6}$, Kerry Lenzi ${ }^{5}$, Ayman Rezk ${ }^{4,5}$, Kristina R. Patterson ${ }^{4,5}$, Diego A. Espinoza ${ }^{4,5,7}$, \\ Jessy C. Kadri, 4,5, Daniel M. Markowitz ${ }^{4,5}$, Clyde E. Markowitz ${ }^{4,5}$, Ina Mexhitaj ${ }^{4,5}$, Dina Jacobs ${ }^{4,5}$, \\ Allison Babb ${ }^{4,5}$, Michael R. Betts ${ }^{1,8}{ }^{1,}$, Eline T. Luning Prak ${ }^{1,9}$, Daniela Weiskopf ${ }^{10}$, Alba Grifoni ${ }^{10}$, \\ Kendall A. Lundgreen ${ }^{8,11}$, Sigrid Gouma ${ }^{1,8}$, Alessandro Sette ${ }^{10,12}$, Paul Bates ${ }^{8,11}$, Scott E. Hensley ${ }^{1,8}$, \\ Allison R. Greenplate ${ }^{1,3}$, E. John Wherry ${ }^{1,3,6,13 凶}$, Rui Li ${ }^{4,5}$ and Amit Bar-Or $\mathbb{B}^{4,5} \bowtie$
}

SARS-CoV-2 messenger RNA vaccination in healthy individuals generates immune protection against COVID-19. However, little is known about SARS-CoV-2 mRNA vaccine-induced responses in immunosuppressed patients. We investigated induction of antigen-specific antibody, B cell and T cell responses longitudinally in patients with multiple sclerosis (MS) on anti-CD20 antibody monotherapy $(n=20)$ compared with healthy controls $(n=10)$ after BNT162b2 or mRNA-1273 mRNA vaccination. Treatment with anti-CD20 monoclonal antibody (aCD20) significantly reduced spike-specific and receptor-binding domain (RBD)-specific antibody and memory B cell responses in most patients, an effect ameliorated with longer duration from last aCD20 treatment and extent of B cell reconstitution. By contrast, all patients with MS treated with aCD20 generated antigen-specific CD4 and CD8 T cell responses after vaccination. Treatment with aCD20 skewed responses, compromising circulating follicular helper $T\left(T_{F H}\right)$ cell responses and augmenting CD8 $T$ cell induction, while preserving type 1 helper $T\left(T_{H}\right)$ cell priming. Patients with MS treated with aCD20 lacking anti-RBD IgG had the most severe defect in circulating $T_{\mathrm{FH}}$ responses and more robust CD8 $T$ cell responses. These data define the nature of the SARS-CoV-2 vaccine-induced immune landscape in aCD20-treated patients and provide insights into coordinated mRNA vaccine-induced immune responses in humans. Our findings have implications for clinical decision-making and public health policy for immunosuppressed patients including those treated with aCD20.

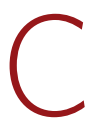
oronavirus disease 19 (COVID-19) has caused a global pandemic with profound public health and socioeconomic sequelae due to the absence of protective immunity to SARS-CoV-2, the viral infectious cause of COVID-19 (refs. ${ }^{1,2}$ ). Vaccines were rapidly developed with the goals of protecting individuals and achieving herd immunity ${ }^{3}$. The two mRNA vaccines granted Food and Drug Administration Emergency Use Authorization in the US, BNT162b2 (Pfizer-BioNTech) and mRNA1273 (Moderna), were shown in phase 3 clinical trials of healthy individuals to be highly effective in preventing moderate-to-severe COVID-19 (refs. ${ }^{4,5}$ ). Individuals with underlying autoimmune disorders, including MS, and those on immune-modulatory therapies were not included in these trials. As a result, the magnitude and quality of the immune response to mRNA vaccination is not well characterized in these potentially vulnerable patients who may be at greater risk for COVID-19-associated morbidity and mortality and more prone to infect others ${ }^{6-1}$

aCD20-based B cell-depleting strategies are implemented in hematological malignancies ${ }^{13}$ and a variety of autoimmune disorders $^{14}$, including $\mathrm{MS}^{15,16}$. On antigen exposure, B cells can form memory B cells or differentiate into plasmablasts and plasma cells ${ }^{17}$. As a result, vaccine-specific antibody responses are diminished in patients on aCD20 therapy ${ }^{18-23}$. For SARS-CoV-2 mRNA vaccination, B cell depletion results in decreased spike-specific antibodies

'Institute for Immunology, University of Pennsylvania Perelman School of Medicine, Philadelphia, PA, USA. ${ }^{2}$ Division of Rheumatology, Department of Medicine, University of Pennsylvania Perelman School of Medicine, Philadelphia, PA, USA. ${ }^{3}$ Immune Health, University of Pennsylvania Perelman School of Medicine, Philadelphia, PA, USA. ${ }^{4}$ Center for Neuroinflammation and Experimental Therapeutics, University of Pennsylvania Perelman School of Medicine, Philadelphia, PA, USA. ${ }^{5}$ Department of Neurology, University of Pennsylvania Perelman School of Medicine, Philadelphia, PA, USA. ${ }^{6}$ Department of Systems Pharmacology and Translational Therapeutics, University of Pennsylvania Perelman School of Medicine, Philadelphia, PA, USA. ${ }^{7}$ Immunology Graduate Group, Perelman School of Medicine, University of Pennsylvania, Philadelphia, PA, USA. ${ }^{8}$ Department of Microbiology, University of Pennsylvania Perelman School of Medicine, Philadelphia, PA, USA. ${ }^{9}$ Department of Pathology and Laboratory Medicine, University of Pennsylvania Perelman School of Medicine, Philadelphia, PA, USA. ${ }^{10}$ Center for Infectious Disease and Vaccine Research, La Jolla Institute for Immunology, La Jolla, CA, USA. "1Penn Center for Research on Coronavirus and Other Emerging Pathogens, University of Pennsylvania Perelman School of Medicine, Philadelphia, PA, USA.

${ }^{12}$ Department of Medicine, Division of Infectious Diseases and Global Public Health, University of California San Diego, La Jolla, CA, USA. ${ }^{13 P a r k e r}$ Institute for Cancer Immunotherapy, University of Pennsylvania Perelman School of Medicine, Philadelphia, PA, USA. ${ }^{14}$ These authors contributed equally: Sokratis A. Apostolidis, Mihir Kakara, Mark M. Painter, Rishi R. Goel. $\varpi_{e}$-mail: wherry@pennmedicine.upenn.edu; lirui158@pennmedicine.upenn.edu; amitbar@pennmedicine.upenn.edu 
in patients with chronic inflammatory disease ${ }^{24}$, including patients with $\mathrm{MS}^{25}$. However, the kinetics of antibody responses and their relationship to peripheral B cell depletion and spike-specific memory B cells are poorly understood.

The role of $\mathrm{B}$ cells in $\mathrm{T}$ cell priming, differentiation and proliferation is unclear, especially in humans. Some studies suggest that $\mathrm{B}$ cells are not required for $\mathrm{T}$ cell responses ${ }^{26-28}$ whereas other work supports a role for B cells as antigen-presenting cells that facilitate $\mathrm{T}$ cell priming ${ }^{29-35}$. In COVID-19, CD 4 and CD8 T cell immunity is generated with $\mathrm{T}$ cell responses correlating with better outcomes in some settings ${ }^{36-38}$. Robust CD8 $\mathrm{T}$ cell responses are associated with improved survival in COVID-19 patients with hematologic malignancies, including patients on therapies that deplete B cells ${ }^{39}$. These data suggest that $\mathrm{T}$ cells may provide protective immunity and limit severe disease in settings where antibody responses are lacking. In addition, $\mathrm{T}$ cells are capable of recognizing mutant SARS-CoV-2 variants ${ }^{40,41}$ that can partially escape humoral-based immunity. Despite these data, the induction of $\mathrm{T}$ cell responses by mRNA vaccination in patients on $\mathrm{B}$ cell-depleting therapies is poorly understood.

In this study, we analyzed patients with MS to evaluate the effect of aCD20 therapy on SARS-CoV-2 mRNA vaccine responses. Although most patients with MS treated with aCD20 (MS-aCD20) made detectable spike-binding antibodies and 50\% made RBD antibodies, antibody titers were lower, delayed and had reduced neutralizing activity compared with healthy controls. All patients with MS treated with aCD20 developed spike-specific CD4 T cell responses and enhanced CD8 T cell responses. Finally, comparing patients with MS treated with aCD20 who did and did not generate anti-RBD IgG responses revealed differences in immune response coordination, with substantial reduction in vaccine-induced circulating $\mathrm{T}_{\mathrm{FH}}$ cell responses and reciprocal increases in CD8 $\mathrm{T}$ cell responses in those who lacked anti-RBD antibodies. These studies provide insights into the role of B cells and humoral immunity in vaccine-induced $\mathrm{T}$ cell responses and shed light on the immune mechanisms that accompany aCD20 therapy based on differential responses to vaccination.

\section{Results}

Impact of aCD20 therapy on mRNA vaccine-induced antibody responses. To examine the effect of aCD20 therapy on responses to SARS-CoV-2 mRNA vaccination, we recruited 20 patients with MS treated with aCD20 monotherapy and compared their vaccine-induced immune responses to 10 healthy controls (Extended Data Fig. 1). All patients with MS and healthy controls had no previous clinical signs or symptoms of COVID-19. Plasma and peripheral blood mononuclear cells (PBMCs) were analyzed at five time points before and after vaccination (Fig. 1a).

All healthy controls generated both anti-spike and anti-RBD IgG after the first dose of mRNA vaccine and antibody increased after the second dose (Fig. 1b,c), as reported ${ }^{42}$. By contrast, responses were more variable in patients with MS treated with aCD20, with $89 \%$ developing detectable anti-spike IgG and only $50 \%$ mounting detectable anti-RBD IgG responses by T5 (Fig. 1b,c and Extended Data Fig. 2). Among those patients with MS treated with aCD20 with detectable IgG, the magnitude was generally lower and the kinetics of the IgG response delayed compared to healthy controls. Moreover, the generation of neutralizing antibody by T4 and T5 was significantly reduced in the MS-aCD20 group (Extended Data Fig. 3a). Neutralizing and binding antibody titers for spike and RBD were correlated for both patients and healthy controls (Extended Data Fig. 3b). These findings extend previous observations ${ }^{24,25}$ that antibody responses to SARS-CoV-2 mRNA vaccine are attenuated in patients with MS on aCD20 therapy.

Because a major reason for the altered antibody responses in patients with MS treated with aCD20 was likely to be depletion of B cells, we considered whether the heterogeneity in antibody responses (Fig. 1b,c) was related to the duration between vaccination and the last aCD20 infusion. There were trends toward increased serologic responses to both spike (Extended Data Fig. 3c) and RBD (Extended Data Fig. 3d) as the duration from the last aCD20 infusion increased. To further test this idea, we quantified $\mathrm{CD} 19^{+} \mathrm{B}$ cell numbers in circulation (Extended Data Fig. 3e). Although most patients with MS treated with aCD20 had no detectable B cells, small circulating B cell populations were observed in some patients and there was a clear relationship between time since last aCD20 infusion and the extent of B cell reconstitution (Fig. 1d). Patients with MS treated with aCD20 with higher percentages of circulating B cells before the vaccine (T1) had more robust anti-spike and anti-RBD IgG responses at T4 and T5 (Fig. 1e), demonstrating a correlation between mRNA vaccine antibody responses and the extent of $\mathrm{B}$ cell reconstitution at the time of vaccination. The small number of patients with MS treated with aCD20 who had circulating B cell frequencies comparable to healthy controls achieved equivalent antibody titers after vaccination (Fig. 1e), which suggests that $\mathrm{B}$ cells repopulating the periphery after aCD20 infusion are functionally competent. Thus, when the circulating B cell pool is repopulated with increased time since last aCD20 administration, vaccine-induced antibody responses approached those observed in healthy controls.

aCD20 effects on vaccine-induced antigen-specific memory $B$ cells. We next used a spike and RBD B cell probe strategy ${ }^{42}$ to define the magnitude and kinetics of the memory $\mathrm{B}$ cell response in patients with MS treated with aCD20 after SARS-CoV-2 mRNA vaccination (Methods). Although circulating memory B cells specific for both spike (Extended Data Fig. 3f and Fig. 1f) and RBD (Extended Data Fig. $3 \mathrm{f}$ and Fig. $1 \mathrm{~g}$ ) were readily induced in all healthy controls, spike-specific memory B cells were detected in only a subset of patients with MS treated with aCD20, where their frequencies were also substantially diminished (Fig. 1f) at all time points (Supplementary Table 1). Similarly, only a minority of patients with MS treated with aCD20 generated detectable RBD-specific memory B cells (Fig. 1g and Supplementary Table 1). Finally, there was a strong correlation between detection of antigen-specific memory $\mathrm{B}$ cells and longer duration since the last aCD20 treatment (Fig. $1 \mathrm{~h}, \mathrm{i}$ ). There were substantially more patients with detectable antibody responses $(88.9 \%)$ than patients with detectable circulating memory B cells $(30 \%)$ to the spike antigen, perhaps suggesting a role for repopulation of $\mathrm{B}$ cells in lymphoid tissues before the blood. Overall, however, these data indicate that memory $\mathrm{B}$ cell responses after SARS-CoV-2 mRNA vaccination were compromised in patients with MS treated with aCD20 compared with healthy controls especially in patients who were immunized in closer proximity to their last aCD20 infusion.

aCD20 impact on vaccine-induced CD4 $\mathrm{T}$ cell responses. The impact of aCD20 treatment on T cell responses to SARS-CoV-2 mRNA vaccination is unclear. To examine this question, we implemented high-dimensional flow cytometry analysis of circulating $\mathrm{T}$ cell populations after SARS-CoV-2 mRNA vaccination, using optimized $t$-distributed stochastic neighbor embedding (opt-SNE) dimensionality reduction followed by FlowSOM clustering (Supplementary Fig. 1 and Extended Data Fig. 4). Examining the total $\mathrm{CD} 4^{+} \mathrm{T}$ cell landscape over time revealed dynamic changes after mRNA vaccination (Extended Data Fig. 4a). The total landscape was mapped with key markers (Extended Data Fig. 4b) and metaclusters corresponding to distinct subpopulations of CD4 $\mathrm{T}$ cells were defined (Extended Data Fig. 4c,d). A group of small metaclusters (metaclusters 9-14) was identified that expanded after the first vaccine dose in healthy controls and expressed high Ki67, CD38, inducible costimulator (ICOS) and human leukocyte 
a

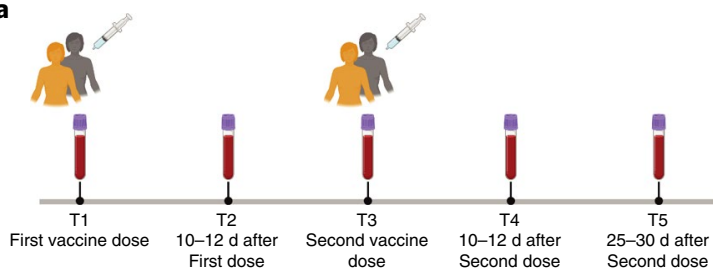

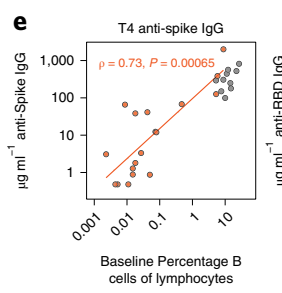

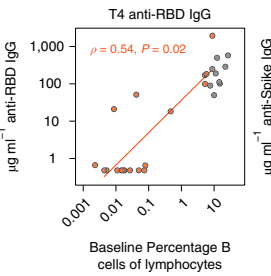

Baseline Percentage
cells of lymphocytes

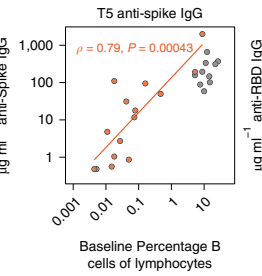

cells of lymphocytes
lecentage

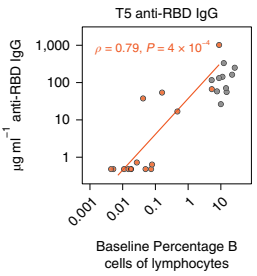

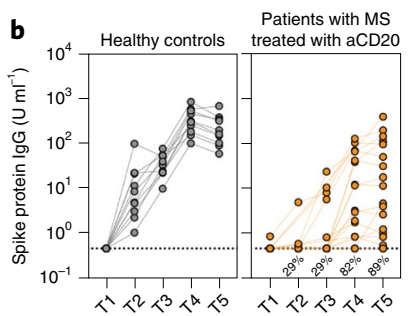
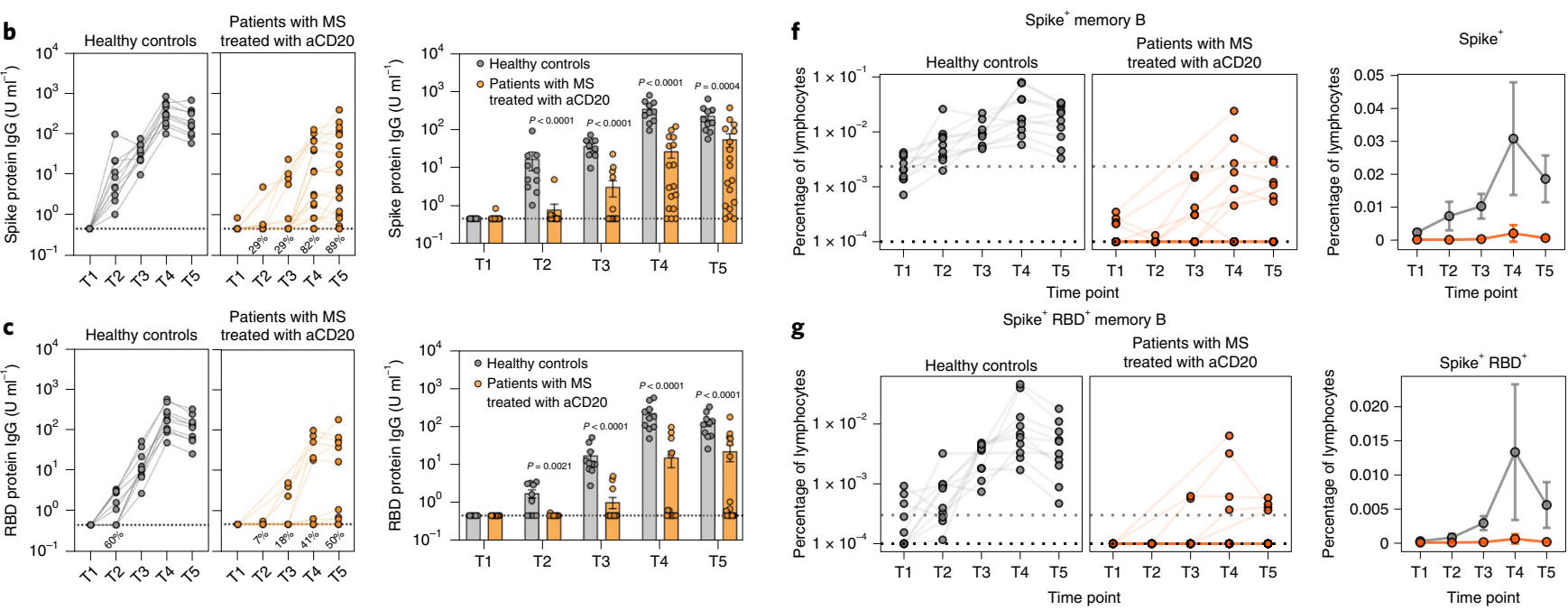

d

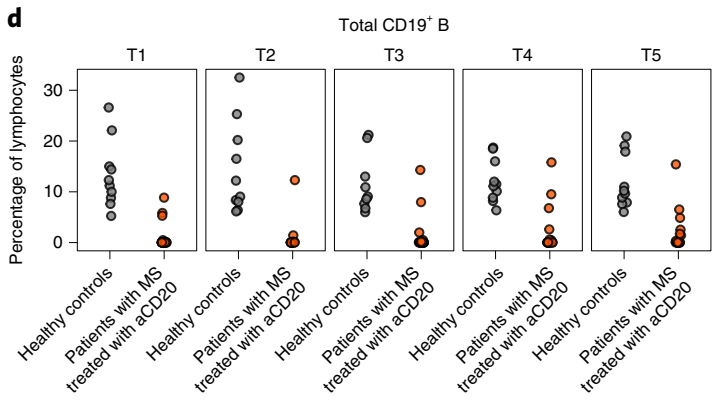

h
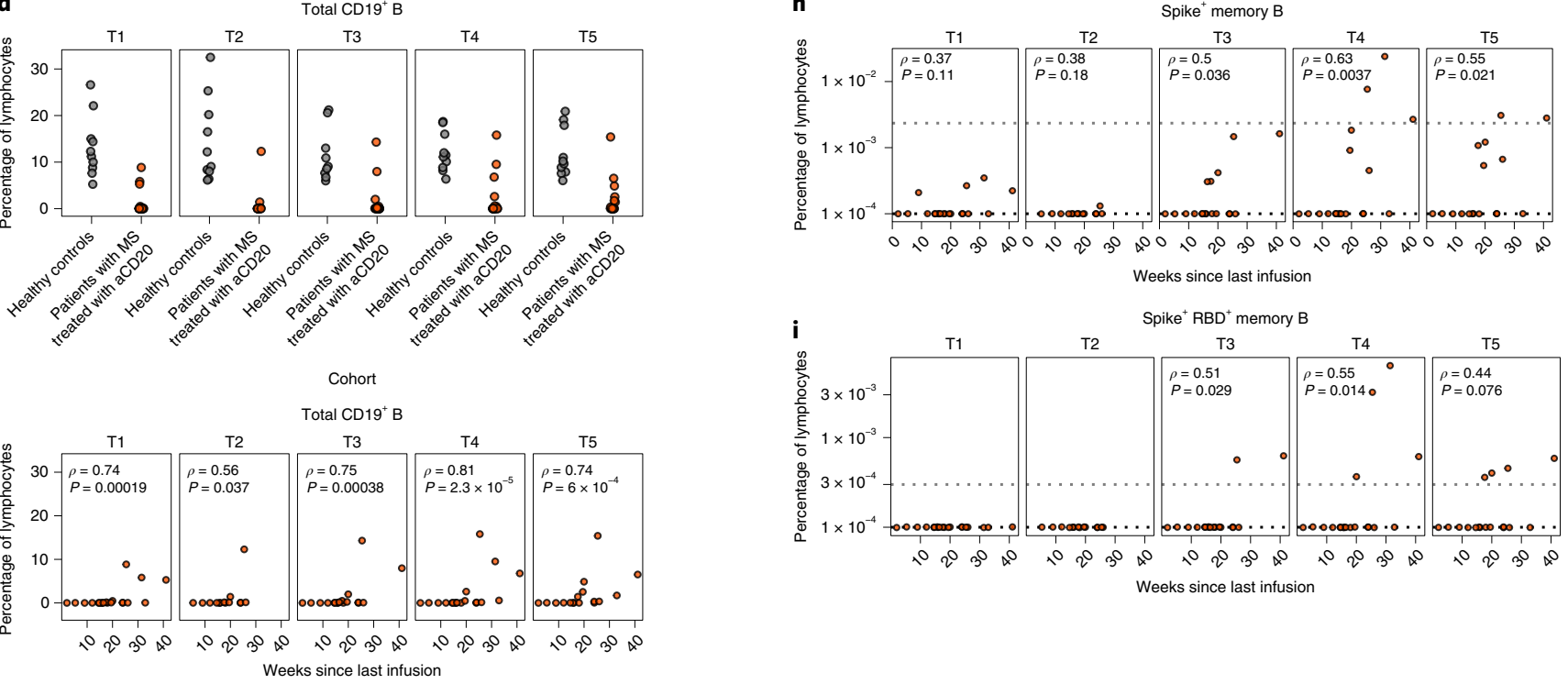

Weeks since last infusio

Fig. 1 | Decreased humoral responses after SARS-CoV-2 mRNA vaccination in patients with MS treated with aCD20. a, Longitudinal study design, vaccine administration scheme and time points collected after SARS-CoV-2 mRNA vaccination for healthy controls and patients with MS treated with aCD20. b,c, Anti-spike IgG (b) and anti-RBD IgG (c) for all time points collected (T1-T5) were measured in healthy controls and patients with MS treated with aCD20. Statistical analysis was performed using an unpaired, two-tailed, nonparametric Wilcoxon test. The bar plots represent the mean \pm s.e.m. d Top: Frequency of CD19+ B cells as a percentage of total lymphocytes in healthy controls and patients with MS treated with aCD20. Bottom: Correlation between the frequency of total CD19+ B cells and weeks since last aCD20 infusion. Correlations were calculated using nonparametric Spearman rank correlation. e, Correlations between the frequency of baseline (T1) percentage of B cells of all lymphocytes and levels of anti-spike IgG or anti-RBD IgG at T4 (left) and T5 (right) after vaccination. Only patients with MS treated with aCD20 were considered for the correlations, with healthy controls as a visual reference. Associations were calculated using Spearman rank correlation and are shown with Pearson trend lines for visualization. $\mathbf{f}, \mathbf{g}$, Frequency of $\operatorname{spike}^{+}(\mathbf{f})$ and spike+RBD+ $(\mathbf{g})$ memory B cells over time in vaccinated individuals. Data are represented as the frequency of antigen-specific cells in the total lymphocyte compartment (left: individual points, log scale; right, mean with $95 \%$ Cls, linear scale). $\mathbf{h}, \mathbf{i}$, Correlation between the frequency of spike+ $(\mathbf{h})$ and spike+RBD+ (i) memory B cells and weeks since last infusion of aCD20. Correlations were calculated using nonparametric Spearman rank correlation. Gray, healthy controls $(n=10)$; orange: patients with MS treated with aCD20 $(n=20)$. 
antigen-DR isotype (HLA-DR), consistent with vaccine-induced activated $\mathrm{T}$ cells. These metaclusters showed less dynamic change in the MS-aCD20 group with more subtle induction at T2 and T4. No differences were observed in the abundance of these metaclusters between the MS-aCD20 and healthy control groups at either T2 or T4 (Extended Data Fig. 4e). We next wanted to gain deeper insights into the CD4 $\mathrm{T}$ cell subpopulations induced by vaccination in MS-aCD20 patients compared with healthy controls.

Vaccination in humans induces $\mathrm{Ki}^{+} 7^{+} \mathrm{CD} 38^{+} \mathrm{CD} 4$ and $\mathrm{CD} 8$ $\mathrm{T}$ cells approximately 1-2 weeks after Se immunization; this activated, proliferating subset contains antigen-specific $\mathrm{T}$ cells ${ }^{43-46}$. Consistent with previous reports ${ }^{47}$, a population of $\mathrm{Ki}^{+} 7^{+} \mathrm{CD} 38^{+}$ CD4 $\mathrm{T}$ cells was induced after the first vaccine dose in healthy controls, peaking at T2 and then returning to baseline (Fig. 2a and Supplementary Fig. 1). Patients with MS treated with aCD20 had similar frequencies of activated CD4 T cells at baseline. However, their $\mathrm{Ki} 67^{+} \mathrm{CD} 38^{+} \mathrm{CD} 4 \mathrm{~T}$ cells were less induced after vaccination compared to healthy controls at T2, displayed no increase after the second dose and remained lower than healthy controls through T5 (Fig. 2a). Comparison of these activated $\mathrm{Ki} 67^{+} \mathrm{CD} 38^{+} \mathrm{CD} 4 \mathrm{~T}$ cells revealed landscape differences independent of vaccination or time point in patients with MS treated with aCD20 versus healthy controls (Fig. 2b). However, there were also clear patterns of vaccine-induced change in subpopulations of $\mathrm{Ki} 67^{+} \mathrm{CD} 38^{+} \mathrm{CD} 4 \mathrm{~T}$ cells. There were areas of more intense Ki67 or CD38 expression, as well as areas of cells that expressed FOXP3, CTLA-4, CXCR5, CXCR3, CCR6, T-bet and other activation markers corresponding to distinct subpopulations of activated CD4 T cells (Fig. 2c-e and Extended Data Fig. 5a). Additional metaclusters were identified with clear enrichment after vaccination. Specifically, metacluster 1 increased at T2 and metacluster 7 increased at both T2 and T4 (Fig. 2f). Metacluster 1 was composed of highly activated $\mathrm{Ki} 7^{++} \mathrm{ICOS}^{++} \mathrm{CXCR}^{+} \mathrm{T}-$ bet $^{\text {mid }}$ CD4 $\mathrm{T}$ cells of the central memory $\left(\mathrm{T}_{\mathrm{CM}}\right)$ /type 1 effector memory $\left(\mathrm{T}_{\mathrm{EM} 1}\right)$ phenotype $\left(\mathrm{T}_{\mathrm{CM}} / \mathrm{T}_{\mathrm{EM} 1} \mathrm{~T}_{\mathrm{H}} 1\right.$ cells). Metacluster 7 represented CCR6 $^{+}$T-bet ${ }^{-}$or CXCR3 $^{+}$T-bet ${ }^{\text {mid }} \mathrm{T}_{\mathrm{CM}} / \mathrm{T}_{\mathrm{EM} 1}$ CD4 $\mathrm{T}$ cells with high ICOS $\left(\mathrm{T}_{\mathrm{CM}} / \mathrm{T}_{\mathrm{EM} 1} \mathrm{~T}_{\mathrm{H}} 17\right.$ - and $\mathrm{T}_{\mathrm{H}} 1$-like cells). The dynamic changes in these two metaclusters after vaccination were similar between the MS-aCD20 and healthy control groups (Fig. 2f). We next sought to understand the response of circulating $\mathrm{T}_{\mathrm{FH}}$ cells given the role of $\mathrm{T}_{\mathrm{FH}}$ cells in supporting antigen-specific $\mathrm{B}$ cell responses. Metacluster 3 was an activated $\left(\mathrm{CD} 38^{+} \mathrm{ICOS}^{+} \mathrm{HLA}-\mathrm{DR}^{+}\right)$, proliferating $\left(\mathrm{Ki}^{2} 7^{+}\right)$ subpopulation with high expression of CXCR5 and PD-1 (Fig. $2 \mathrm{e}$ ), corresponding to activated circulating $\mathrm{T}_{\mathrm{FH}}$ cells. This metacluster was similarly induced after the first vaccine dose for both patients with MS treated with aCD20 and healthy controls (Fig. $2 f$ and Extended Data Fig. 5b). However, after the second vaccine dose and through T5, metacluster 3 decreased in proportion (Fig. 2f) and contracted (Extended Data Fig. 5b) in patients with MS treated with aCD20 compared to healthy controls. Thus, this analysis identified subpopulations of CD4 $\mathrm{T}$ cells that responded similarly to vaccination when comparing patients with MS treated with aCD20 to healthy controls (for example, subsets of activated $T_{H} 1$ cells) as well as circulating $\mathrm{T}_{\mathrm{FH}}$ cells that had similar initial induction in the two cohorts but poor maintenance in patients with MS treated with aCD20.

To examine bona fide antigen-specific CD4 T cell responses, we performed spike peptide-dependent activation-induced marker (AIM) assays (Methods). $\mathrm{AIM}^{+} \mathrm{CD}^{+} \mathrm{T}$ cells were defined by coexpression of CD200 and CD40L (Fig. 3a and Extended Data Fig. 6a). The absence of $B$ cells during the AIM peptide stimulation assay did not impact this assay (Extended Data Fig. 6b). After the first dose of the SARS-CoV-2 mRNA vaccine, $\mathrm{AIM}^{+} \mathrm{CD} 4 \mathrm{~T}$ cells were robustly increased in MS-aCD20 and healthy controls, indicating efficient CD4 T cell priming (Fig. 3b). Healthy controls retained high $\mathrm{AIM}^{+} \mathrm{CD} 4 \mathrm{~T}$ cell frequencies at all subsequent time points with a trend toward an additional increase after the second vaccine dose (Fig. 3b), which is consistent with our previous studies ${ }^{47}$. $\mathrm{AIM}^{+} \mathrm{CD} 4$ $\mathrm{T}$ cell responses in patients with MS treated with aCD20 were comparable to healthy controls at all time points examined (Fig. 3b). To further assess memory $\mathrm{T}$ cell subsets, we subdivided $\mathrm{AIM}^{+} \mathrm{CD} 4$ $\mathrm{T}$ cells into $\mathrm{T}_{\mathrm{CM}}$, three different subpopulations of effector memory $\mathrm{T}$ cells $\left(\mathrm{T}_{\mathrm{EM} 1}, \mathrm{~T}_{\mathrm{EM} 2}, \mathrm{~T}_{\mathrm{EM} 3}\right)$ and $\mathrm{T}_{\mathrm{EM}}$ cells reexpressing CD45RA $\left(\mathrm{T}_{\mathrm{EMRA}}\right)$ (Fig. $3 \mathrm{c}$ and Extended Data Fig. 6c). There were no major differences in the distribution of $\mathrm{AIM}^{+} \mathrm{CD} 4 \mathrm{~T}$ cells among these memory $\mathrm{T}$ cell subsets between patients with MS treated with aCD20 and healthy controls, with most $\mathrm{AIM}^{+} \mathrm{CD} 4 \mathrm{~T}$ cells mapping to the $\mathrm{T}_{\mathrm{CM}}$ and $\mathrm{T}_{\mathrm{EM} 1}$ subsets (Fig. 3d) in both groups. Similarly, we used CXCR5, CXCR3 and CCR6 (Fig. 3e and Extended Data Fig. 6d) to examine CD4 $\mathrm{T}_{\mathrm{H}}$ cell subsets. Although the distribution was largely similar between the cohorts, there was a trend toward a lower frequency of circulating $\mathrm{T}_{\mathrm{FH}}$ cells among the total $\mathrm{AIM}^{+}$-responding $\mathrm{CD} 4 \mathrm{~T}$ cells in the MS-aCD20 group (Fig. 3f and Extended Data Fig. 6e). Thus, these data indicate that patients with MS treated with aCD20 were capable of generating robust antigen-specific CD4 T cell responses to both vaccine doses despite attenuated antibody responses.

aCD20 impact on vaccine-induced CD8 $\mathrm{T}$ cell responses. We next examined CD8 $\mathrm{T}$ cell responses after vaccination in patients with MS treated with aCD20 and healthy controls. We first assessed activated $\mathrm{Ki} 67^{+} \mathrm{CD} 38^{+} \mathrm{CD} 8 \mathrm{~T}$ cells (Fig. 4) as above for $\mathrm{CD} 4 \mathrm{~T}$ cells. Activated CD8 $\mathrm{T}$ cells moderately expanded after the first vaccine dose in both cohorts, although the magnitude of increase was more robust for healthy controls (Fig. 4a), possibly due to higher pre vaccination (T1) CD8 T cell activation in the MS-aCD20 group. However, patients with MS treated with aCD20 generated a considerably stronger response to the second vaccine dose than the healthy control group. We next applied the metaclustering approach described above for CD4 $\mathrm{T}$ cells to interrogate the differentiation state of the vaccine-responding activated $\mathrm{Ki} 67^{+} \mathrm{CD} 38^{+} \mathrm{CD} 8 \mathrm{~T}$ cells (Fig. 4b-f and Extended Data Figure 7). The opt-SNE landscape map of activated CD8 T cells revealed differences between patients with MS treated with aCD20 and healthy controls before vaccination, including an abundance of $\mathrm{CD} 27^{+} \mathrm{ICOS}{ }^{+} \mathrm{CD} 38^{+} \mathrm{CD} 8 \mathrm{~T}$ cells largely lacking T-bet in patients with MS treated with aCD20 in contrast to $\mathrm{CD} 27^{-}{ }^{-}$-bet ${ }^{+}$CD8 $\mathrm{T}$ cells in healthy controls (Fig. 4b). However, the activated CD8 $\mathrm{T}$ cell populations in both patients and healthy

Fig. 2 | SARS-CoV-2 mRNA vaccination results in altered CD4 T cell activation in patients with MS treated with aCD20. a, The frequency of activated $\mathrm{Ki} 67^{+} \mathrm{CD} 38^{+} \mathrm{CD} 4 \mathrm{~T}$ cells of total non-naive CD4 T cells. Top: Individuals (points) and the mean (thicker line) are shown for each group. Bottom: Tukey box plots (median, Q1 and Q3 quartiles) for each time point and group are depicted. An unpaired, two-tailed Wilcoxon test was used to compare the two groups at each time point (shown under the box plots) or the groups between the time points indicated (shown above the box plots). NS, not significant. b, Opt-SNE projections of concatenated cytometry data for activated Ki67+CD38+ CD4 T cells for each time point and group combination are shown. c, Surface expression intensity of the indicated markers projected on the opt-SNE two-dimensional (2D) map generated with all samples in $\mathbf{b}$ (color scale: mean fluorescence intensity (MFI) expression of each individual marker in a log scale). d, FlowSOM metaclusters were created using activated $\mathrm{Ki} 67{ }^{+} \mathrm{CD} 38+\mathrm{CD} 4 \mathrm{~T}$ cells concatenated from all samples and projected to the opt-SNE map. e, Surface expression intensity heatmap of the markers indicated for each of the ten FlowSOM metaclusters in $\mathbf{d}$ (color scale: row-adjusted $z$-score expression for each individual marker). $\mathbf{f}$, Abundance of metaclusters 1,7 and 3 as the percentage of activated Ki67 ${ }^{+}$CD $38+C D 4$ T cells. Unpaired, two-tailed Wilcoxon test $P$ values are shown when $P<0.05$ between groups. Gray, healthy controls $(n=10)$; orange, patients with MS treated with aCD20 $(n=20) .{ }^{\star} P<0.05,{ }^{\star \star} P<0.01$. 
controls reoriented after each vaccine dose, such that they occupied a similar opt-SNE space (Fig. 4b). Metaclusters defined these vaccine-induced changes (Fig. $4 \mathrm{~d}-\mathrm{f}$ and Extended Data Figure 7 ). Specifically, metacluster 7 and 8 were the main vaccine-responding CD8 $\mathrm{T}$ cell populations in both groups after the first vaccine dose (Fig. 4b,f), representing $\mathrm{T}_{\mathrm{EM} 1}$ CD8 $\mathrm{T}$ cells with high T-bet, ICOS and
CXCR3. Metacluster 8 expressed high HLA-DR, CD38 and PD-1 and comprised a larger fraction of the activated CD8 T cell pool at T2 compared to metacluster 7 (Fig. 4e,f). Metacluster 6 was the predominant population enriched after the second vaccine dose in both groups (Fig. 4b,f). Like metaclusters 7 and 8 , metacluster 6 was a $\mathrm{T}_{\mathrm{EM} 1}$ subset that expressed T-bet. However, metacluster 6 expressed
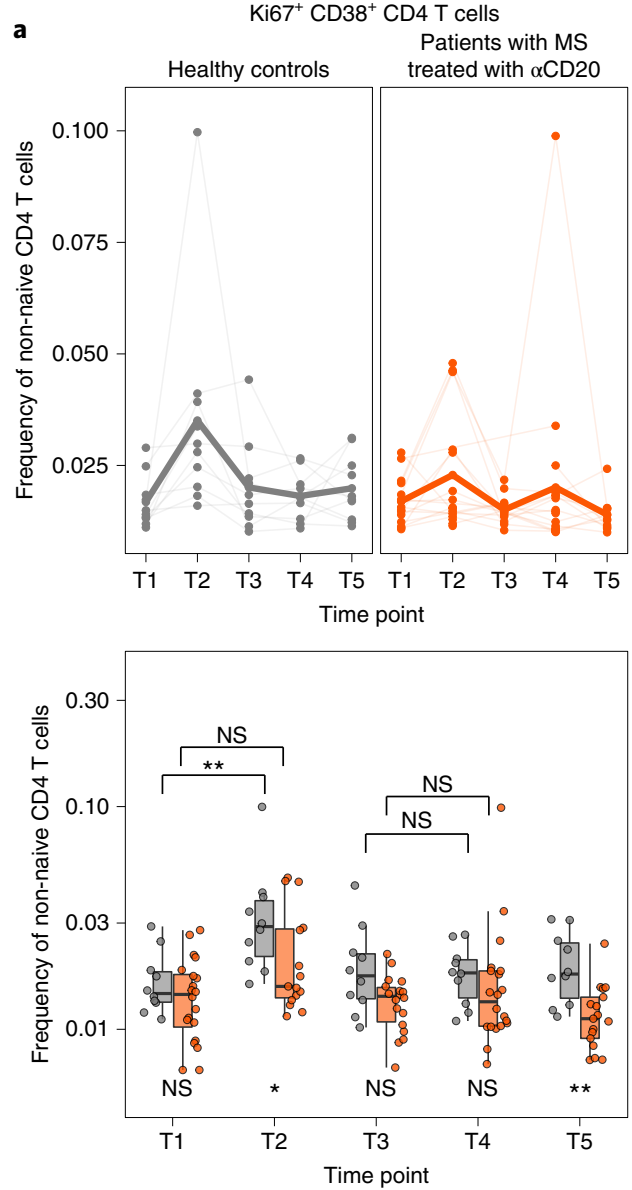

d

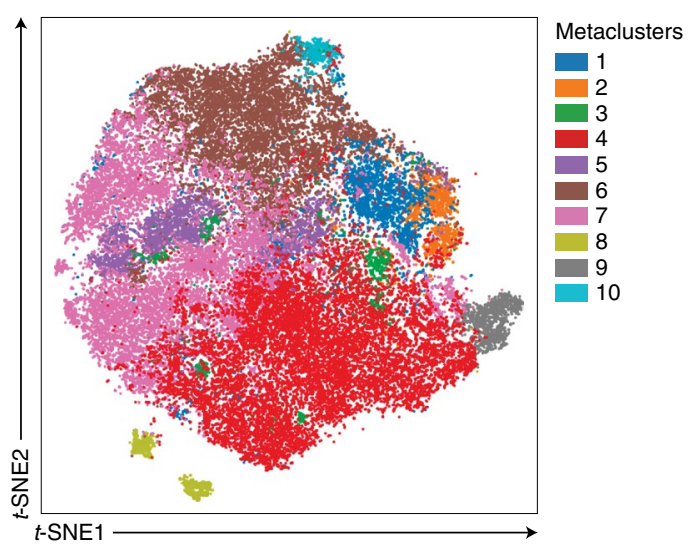

b
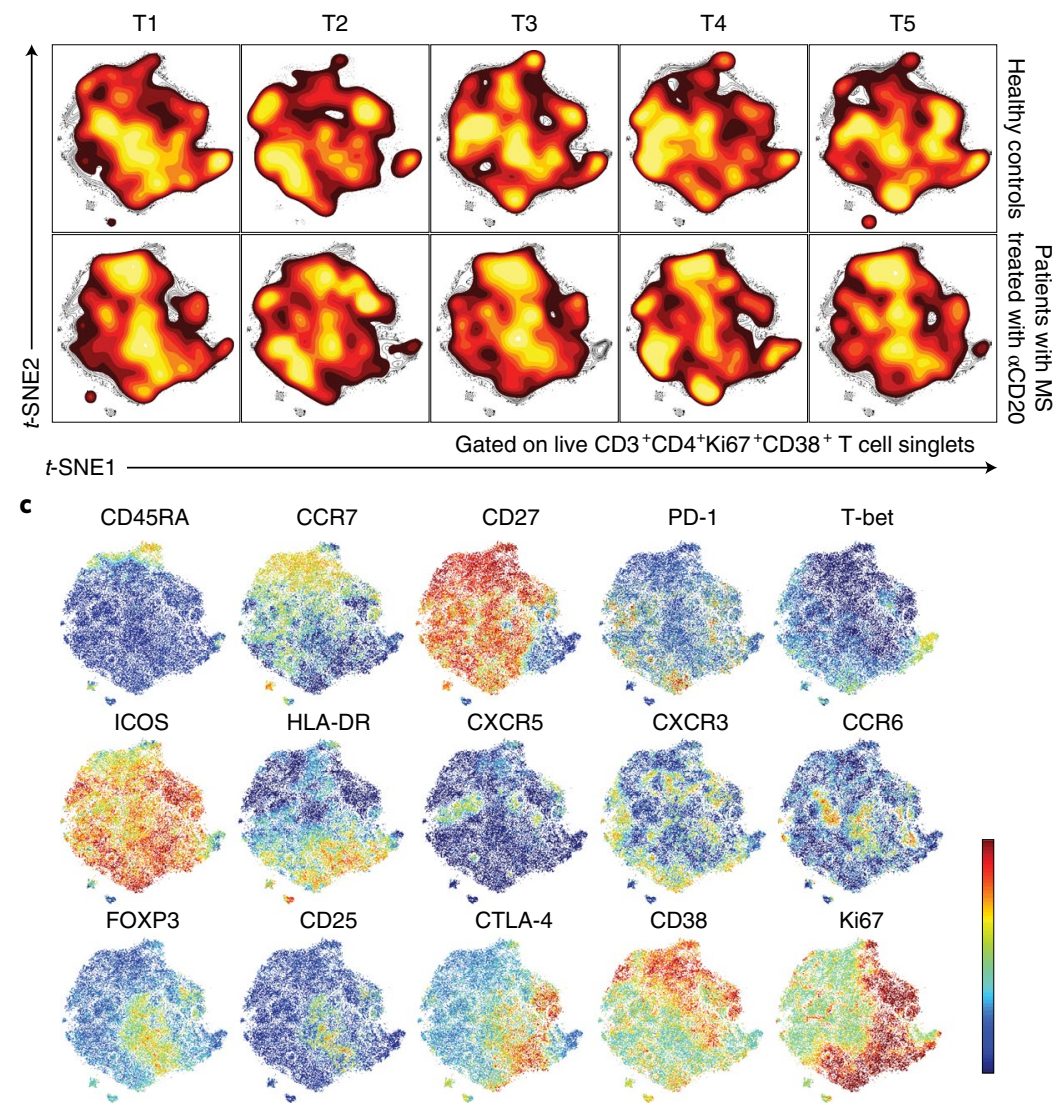

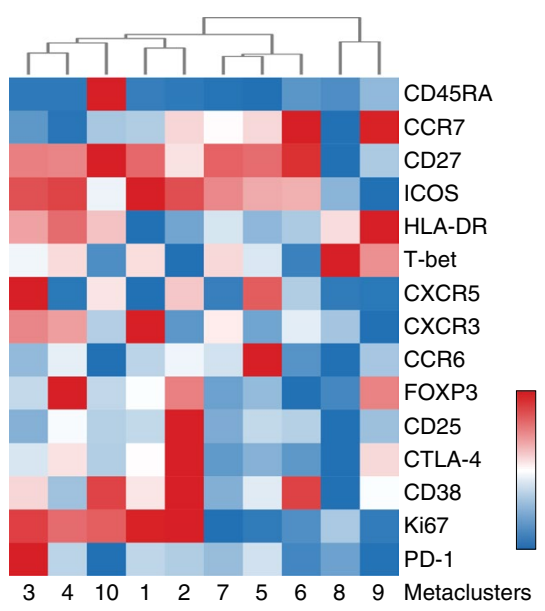

f
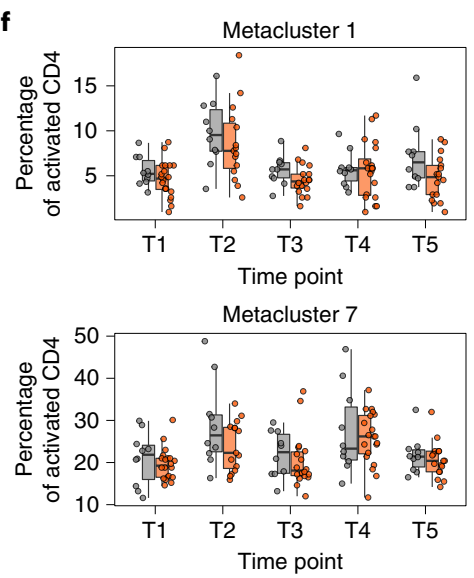

Metacluster 3

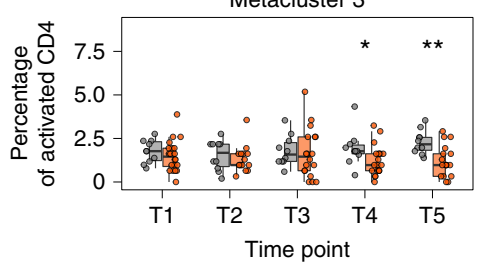


a

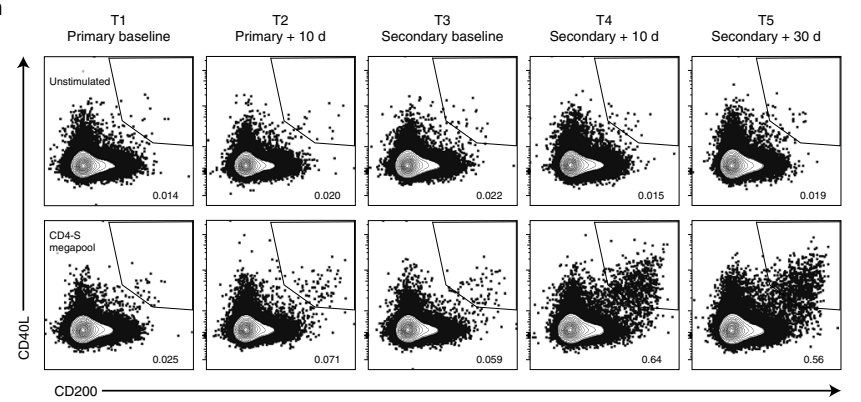

b

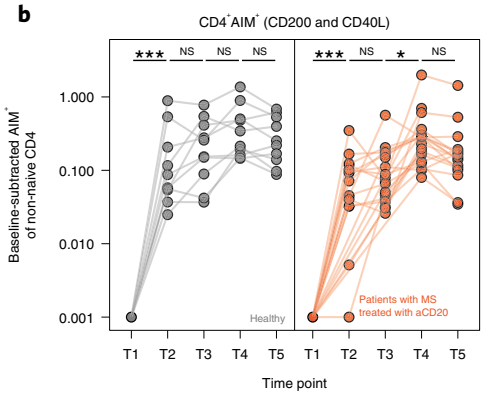

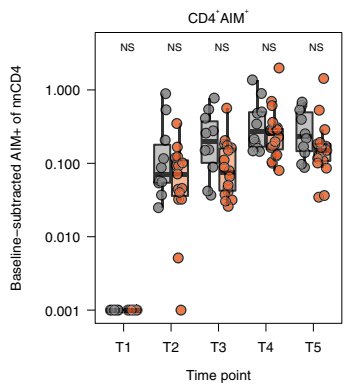

d
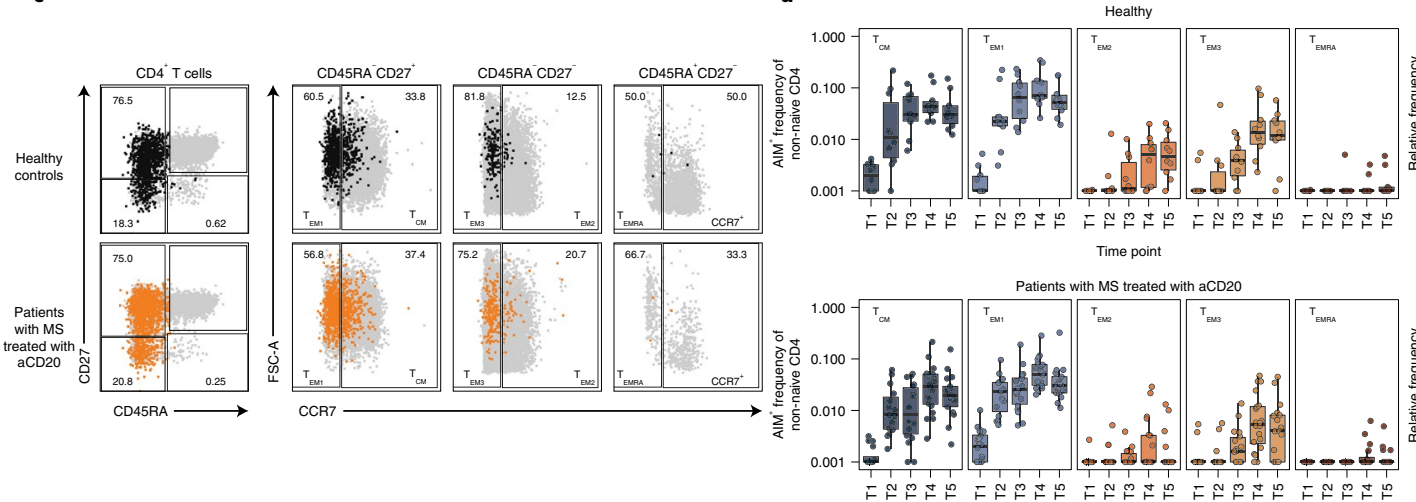

Time point

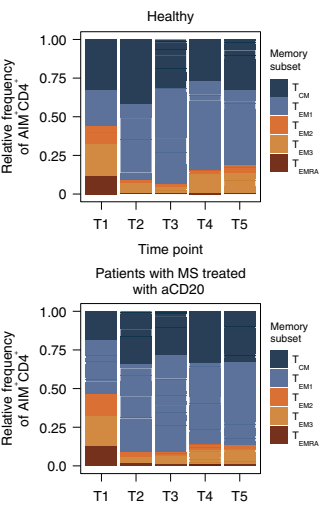

Time point
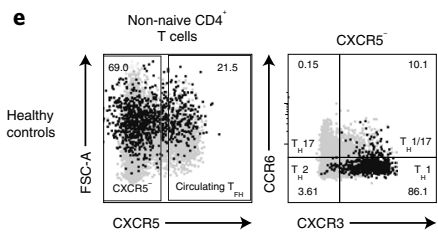

f
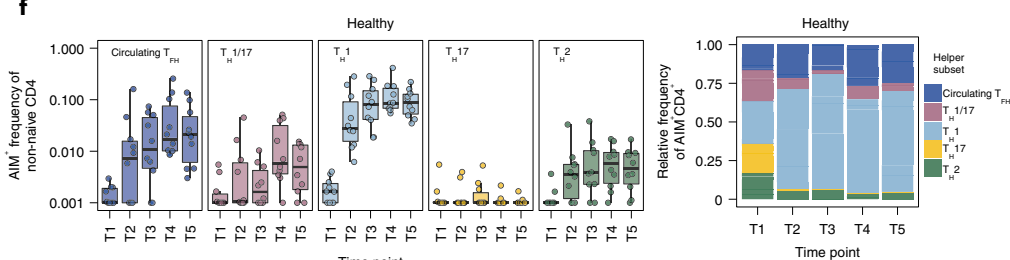

Time point
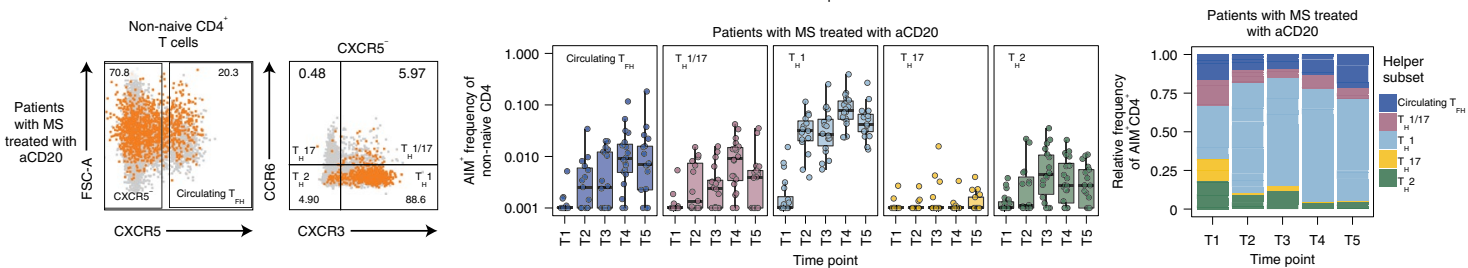

Fig. 3 | Vaccine-specific CD4 T cell responses are comparable between patients with MS treated with aCD20 and healthy controls. a, Representative flow cytometry plots for the quantification of $\mathrm{AIM}^{+} \mathrm{CD} 4 \mathrm{~T}$ cells. The numbers represent the percentage of total non-naive CD4 T cells that are AIM ${ }^{+}$. Top row: Unstimulated. Bottom row: Stimulated with the CD4-S megapool. b, Summary data of AIM+CD4 T cell frequency after vaccination. The values represent the background-subtracted frequency of $\mathrm{AIM}^{+}$non-naive CD4 T cells above paired background-subtracted baseline frequencies. The lines connect individual donors sampled longitudinally. Statistics were calculated using an unpaired, two-tailed Wilcoxon test. Gray, healthy controls ( $n=10)$; orange, patients with MS treated with aCD2O $(n=20)$. c, Representative plots demonstrating the identification of the indicated memory T cell subsets from AIM ${ }^{+}$CD4 T cells. The black or orange events depict $\mathrm{AIM}^{+}$cells from healthy controls or patients with MS treated with aCD20, respectively. The gray events depict the total CD4 T cells from the same donor. The numbers indicate the frequency of AIM+ cells within each gate. $\mathbf{d}$, Frequency of memory T cell subsets in AIM+CD4 T cells. Top: Healthy controls. Bottom: Patients with MS treated with aCD20. Left: Background-subtracted percentage of non-naive $T$ cells that are $\mathrm{AIM}^{+}$cells of each subset. Right: Relative frequency of each memory $\mathrm{T}$ cell subset in the background-subtracted

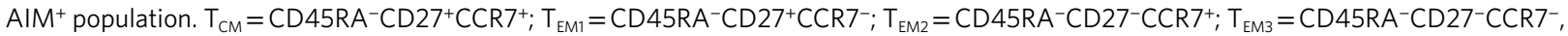

$\mathrm{T}_{\mathrm{EMRA}}=\mathrm{CD} 45 \mathrm{RA}^{+} \mathrm{CD} 27^{-} \mathrm{CCR7}{ }^{-}$. e, Representative flow cytometry plots depicting the gating of $\mathrm{AIM}^{+} \mathrm{CD} 4 \mathrm{~T}$ cells to identify the indicated helper subsets as in c. f, Frequency of helper $T$ cell subsets in $\mathrm{AIM}^{+} \mathrm{CD} 4 \mathrm{~T}$ cells as in $\mathbf{d}$. Circulating $\mathrm{T}_{\mathrm{FH}}=\mathrm{CXCR}^{+}$of non-naive $\mathrm{CD} 4 \mathrm{~T}_{\text {cells; }} \mathrm{T}_{\mathrm{H}} 1=\mathrm{CXCR}^{-} \mathrm{CXCR}^{+} \mathrm{CCR} 6^{-}$;

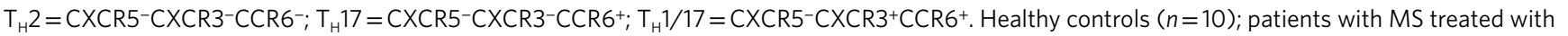
aCD20 $(n=20) .{ }^{\star} P<0.05,{ }^{\star \star} P<0.01,{ }^{\star \star \star} P<0.001$.

intermediate or high HLA-DR and PD-1 but lower ICOS, CXCR3 and CD38 compared to metaclusters 7 and 8 (Fig. 4e). Thus, these high-dimensional cytometry data indicated that vaccine-induced activated CD8 $\mathrm{T}$ cell responses were more robust in patients with MS treated with aCD20 compared to healthy controls after the second vaccine dose. Moreover, the predominant responding CD8 T cell subsets were $\mathrm{T}$-bet ${ }^{+} \mathrm{T}_{\mathrm{EM} 1}$ cells with variable levels of activation and CXCR3 expression.

We next examined antigen-specific CD8 T cell responses after vaccination using spike-dependent AIM assays (Fig. 5). As reported ${ }^{47}$, 


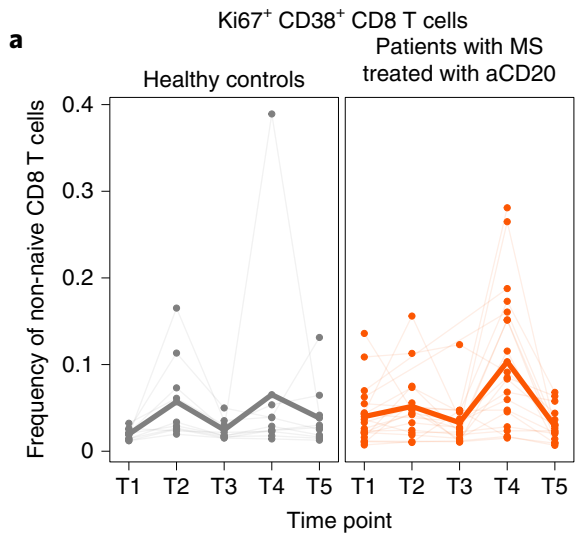

b
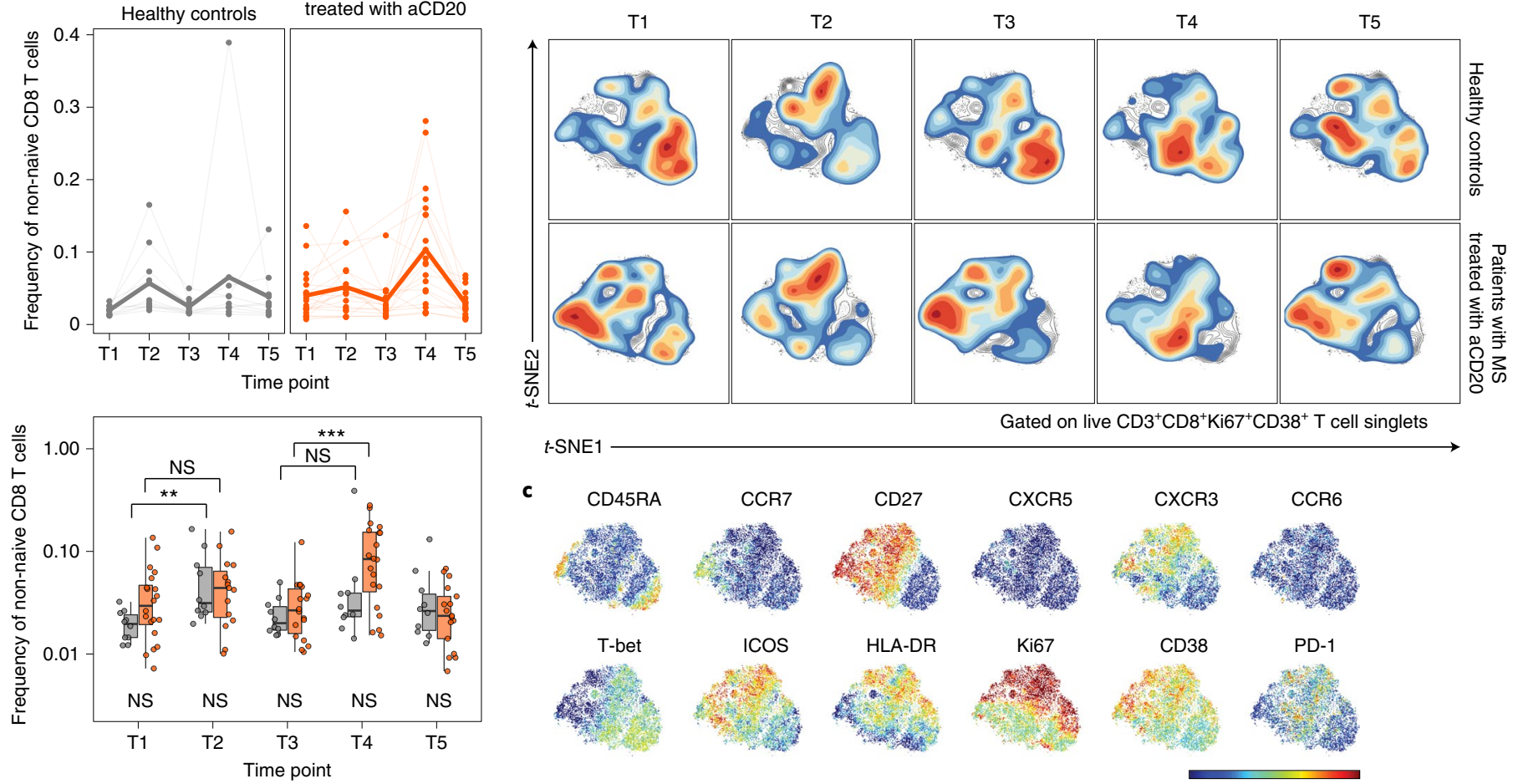

$t-\mathrm{SNE}$

C
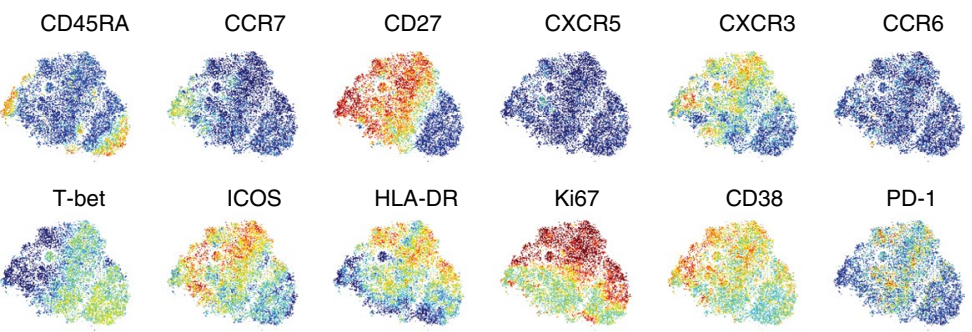

PD-1
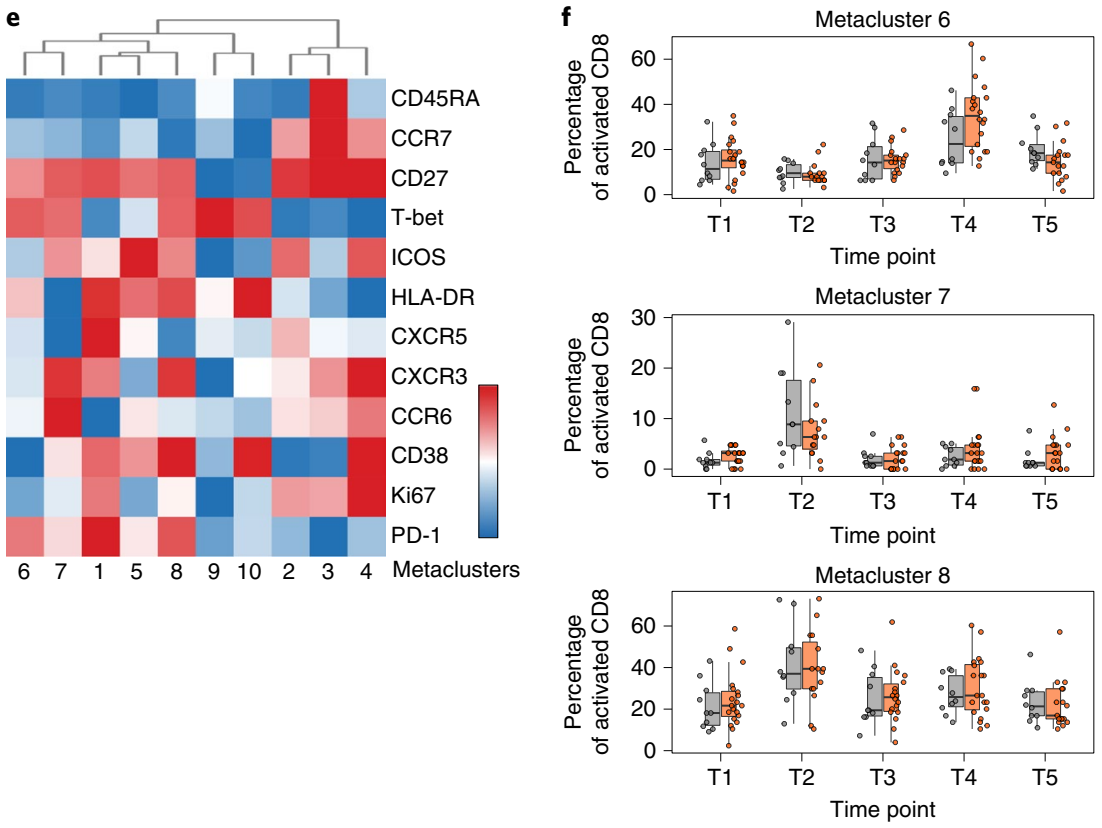

Fig. 4 | SARS-CoV-2 mRNA vaccination results in robust CD8 T cell activation in healthy controls and patients with MS treated with aCD20.

a, Frequency of activated $\mathrm{Ki} 67^{+} \mathrm{CD} 38+\mathrm{CD} 8 \mathrm{~T}$ cells of total non-naive CD8 T cells. Top: Individuals (points) and the mean (thicker line) are shown for each group. Bottom: Tukey box plots (median, Q1 and Q3 quartiles) for each time point and group are depicted. An unpaired, two-tailed Wilcoxon test was used to compare the two groups at each time point (shown under the box plots) or the groups between time points indicated (shown above the box plots). $\mathbf{b}$, The opt-SNE projections of concatenated cytometry data for activated Ki67+CD38+CD8 T cells for each time point and group combination are shown. c, Surface expression intensity of the indicated markers projected on the opt-SNE 2D map generated with all samples in b (color scale: MFI expression of each individual marker in a log scale). d, FlowSOM metaclusters were created using activated Ki67 ${ }^{+}$CD38 ${ }^{+}$CD8 T cells concatenated from all samples and projected onto the opt-SNE map. e, Surface expression intensity heatmap of the markers indicated for each of the ten FlowSOM metaclusters in $\mathbf{d}$ (color scale: row-adjusted $z$-score expression for each individual marker). $\mathbf{f}$, The abundance of metaclusters 6 , 7 and 8 as the percentage of activated $\mathrm{Ki} 67{ }^{+} \mathrm{CD} 38{ }^{+} \mathrm{CD} 8 \mathrm{~T}$ cells is shown for healthy controls and patients with MS treated with aCD20. Unpaired, two-tailed Wilcoxon test $P$ values are shown when $P<0.05$ between groups. Gray, healthy controls $(n=10)$; orange: patients with MS treated with aCD20 $(n=20) .{ }^{\star} P<0.05,{ }^{\star \star} P<0.01,{ }^{\star \star \star} P<0.001$ 


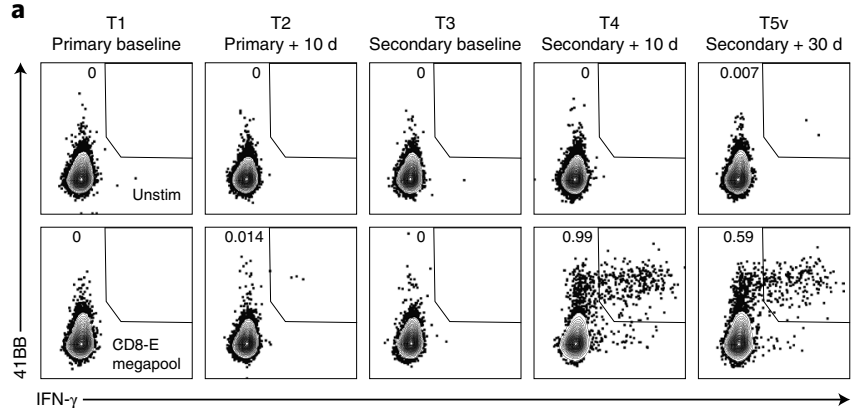

b

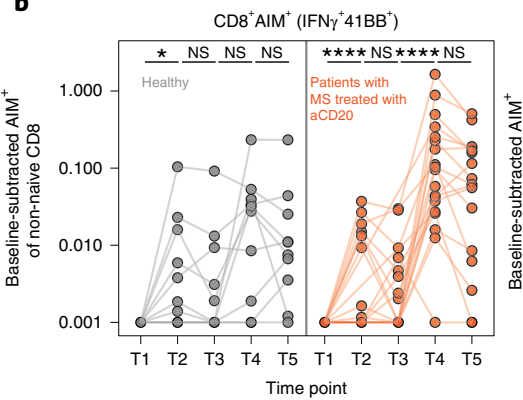

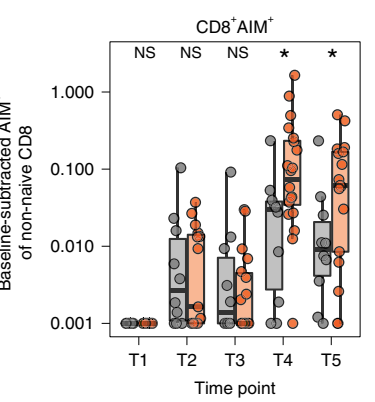

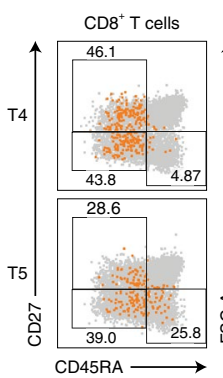

d
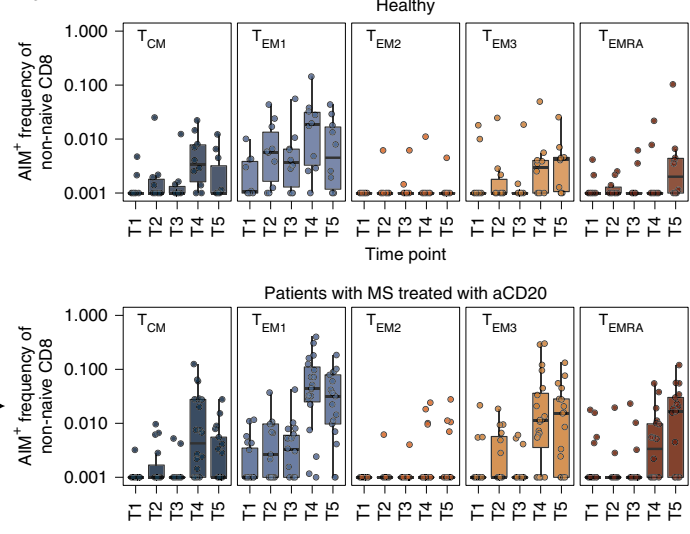

Time point

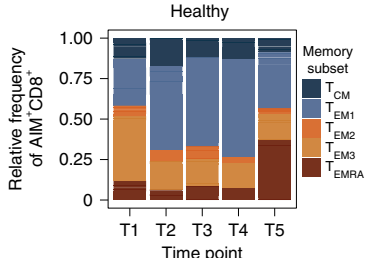

Time point Patients with MS treated
with aCD20 Time point

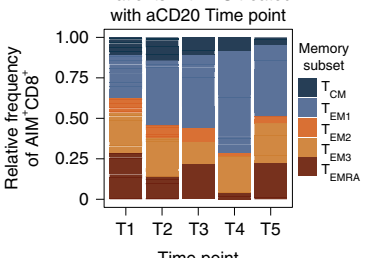

Time point

Fig. 5 | Enhanced antigen-specific CD8 T cell responses after mRNA vaccination in patients with MS treated with aCD20. a, Representative flow cytometry plots for quantifying AIM ${ }^{+} \mathrm{CD} 8 \mathrm{~T}$ cells. The numbers represent the percentage of total non-naive CD8 T cells that are AIM ${ }^{+}$. Top: Unstimulated. Bottom: Stimulated with the CD8-E megapool. b, Summary data of AIM+CD8 T cell frequency after vaccination. The values represent the background-subtracted frequency of $\mathrm{AIM}^{+}$non-naive CD8 T cells above paired background-subtracted baseline frequencies. The lines connect individual donors sampled longitudinally. Statistics were calculated using an unpaired, two-tailed Wilcoxon test. Gray, healthy controls; orange: patients with MS treated with $\mathrm{CCD} 20$. c, Representative flow cytometry plots depicting the identification of the indicated memory T cell subsets from $A I M+C D 8$ $\mathrm{T}$ cells at T4 and T5. The orange events depict AIM+ cells from patients with MS treated with aCD20. The gray events depict the total CD8 T cells from the same donor. The numbers indicate the frequency of $\mathrm{AIM}^{+}$cells within each gate. $\mathbf{d}$, Frequency of memory $\mathrm{T}$ cell subsets in AIM+CD8 $\mathrm{T}$ cells. Top: Healthy controls. Bottom: Patients with MS treated with aCD20. Left: Background-subtracted percentage of non-naive T cells that are AIM+ cells of each subset. Right: Relative frequency of each memory $T$ cell subset in the background-subtracted $\mathrm{AIM}^{+}$population. $\mathrm{T}_{\mathrm{CM}}=\mathrm{CD}^{-} 5 \mathrm{RA}{ }^{-} \mathrm{CD} 27^{+} \mathrm{CCR} 7^{+}$;

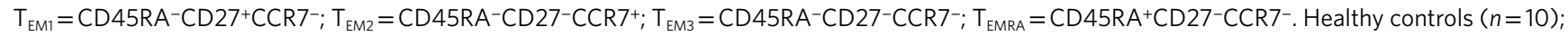
patients with MS treated with aCD20 $(n=20) .{ }^{\star} P<0.05,{ }^{\star \star} P<0.01,{ }^{\star \star \star} P<0.001,{ }^{\star \star \star \star} P<0.0001$.

$\mathrm{AIM}^{+} \mathrm{CD} 8 \mathrm{~T}$ cell responses were detected in a subset of healthy controls after the first vaccine dose, with more individuals responding after the second vaccine dose (Fig. 5a,b). A similar pattern was observed in the patients with MS treated with aCD20. However, after the second vaccine dose (T4), a significantly greater expansion of antigen-specific CD8 T cells was noted in patients with MS treated with aCD20 compared to healthy controls, a difference that persisted at $\mathrm{T} 5$. This expansion was dominated by $\mathrm{T}_{\mathrm{EM} 1}$ cells (Fig. 5 c,d) consistent with the observations above. Of note, both groups had equivalent frequencies of total memory subsets that were largely unchanged by vaccination (Extended Data Figure 8). To assess the functionality of vaccine-specific CD8 T cells, we evaluated the expression of interferon- $\gamma$ (IFN $\gamma$ ), tumor necrosis factor (TNF), interleukin-2 (IL-2) and granzyme B in $\mathrm{AIM}^{+} \mathrm{CD} 8 \mathrm{~T}$ cells at $\mathrm{T} 4$, the peak of the response (Extended Data Figure 9a-c). CD8 T cells at T4 were similarly capable of producing these effector molecules in the healthy control and MS-aCD20 groups whereas there was little granzyme B expression or antigen-specific cytokine production at $\mathrm{T} 1$ before vaccination. Taken together, these data demonstrate that although the overall distribution of memory CD8 $\mathrm{T}$ cell subsets was similar, SARS-CoV-2 mRNA vaccination induced a more robust antigen-specific CD8 $\mathrm{T}$ cell response in patients with MS treated with aCD20 compared to healthy controls, in particular after the second dose of the vaccine.
MS-aCD20 subgroups with distinct vaccine-induced immune coordination. We next examined how variation in the extent of $B$ cell depletion might impact coordination with other features of vaccine-induced immune responses in patients with MS treated with aCD20. First, comparison of antigen-specific measures across $\mathrm{T} 2, \mathrm{~T} 4$ and $\mathrm{T} 5$ revealed a strong correlation between humoral and circulating $\mathrm{T}_{\mathrm{FH}}$ responses (Fig. 6a,b). This correlation was evident earlier and to a stronger extent in the MS-aCD20 group. By contrast, $\mathrm{AIM}^{+} \mathrm{CD} 8 \mathrm{~T}$ cells showed a strong negative correlation with humoral immune features at T5 in the MS-aCD20 group (Fig. 6a,c). $\mathrm{AIM}^{+} \mathrm{T}_{\mathrm{H}} 1$ cells were also no longer positively associated with some features of humoral immunity as observed in healthy controls (Fig. 6a). These findings prompted us to separate patients with MS treated with aCD20 into those who made a detectable RBD-specific IgG response (RBD antibody $\left.{ }^{+}, n=10\right)$ and those who never developed an RBD-specific IgG response (RBD antibody-, $n=10)$, and then investigate other potential immune differences between these two subgroups of patients. Figure $6 \mathrm{~d}$ shows the opt-SNE projections of $\mathrm{Ki} 67^{+} \mathrm{CD} 38^{+} \mathrm{CD} 4 \mathrm{~T}$ cells for the three groups: healthy controls; MS-aCD20 RBD antibody ${ }^{+}$; and MS-aCD20 RBD antibody ${ }^{-}$. The landscape of $\mathrm{Ki} 67^{+} \mathrm{CD} 38^{+} \mathrm{CD} 4 \mathrm{~T}$ cells from RBD antibody ${ }^{+}$ MS-aCD20 patients was similar to that of healthy controls and both RBD antibody ${ }^{+}$MS-aCD20 and healthy controls displayed 


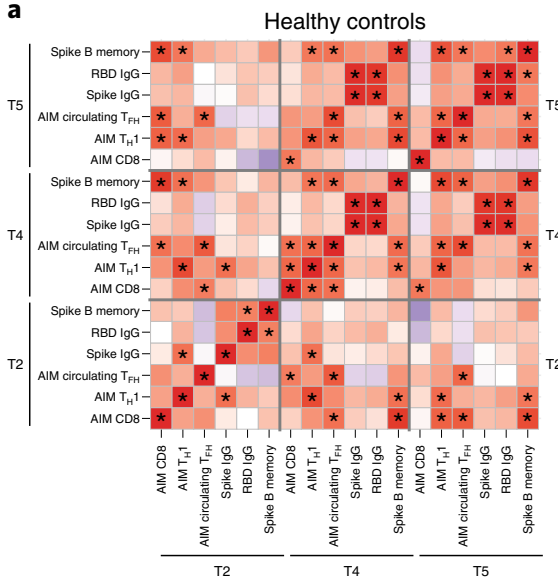

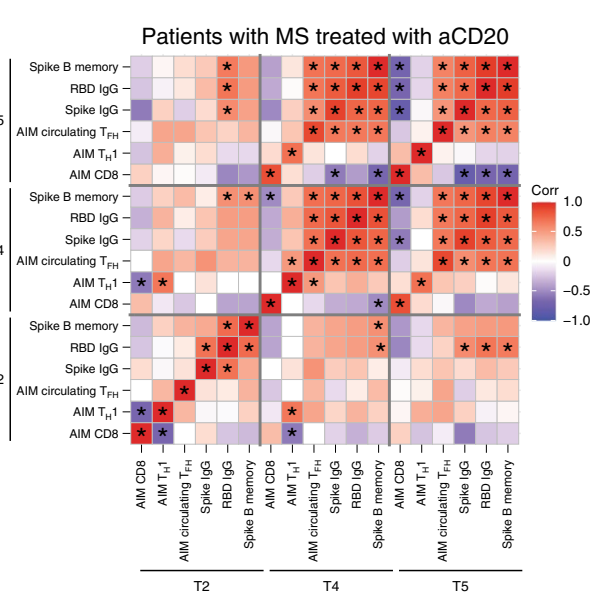
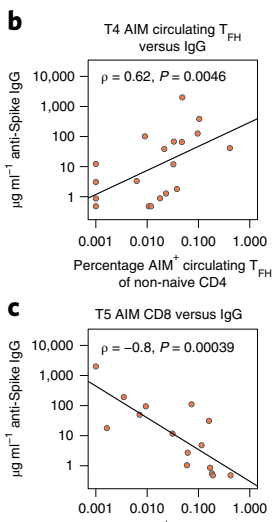

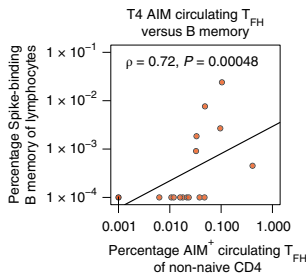

T5 AIM CD8 versus B menty

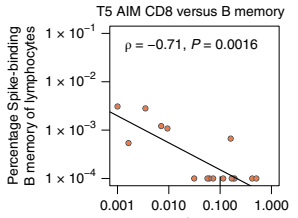

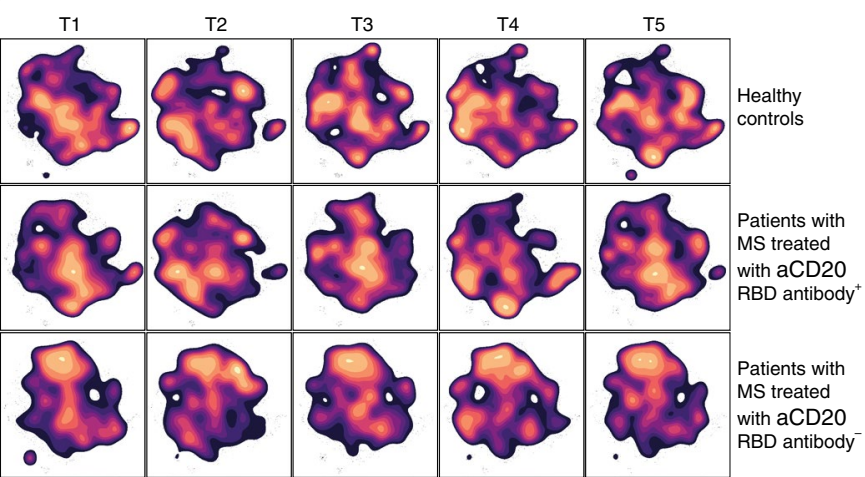

Gated on live $\mathrm{CD}^{+}{ }^{\mathrm{CD}} 4^{+} \mathrm{Ki} 67^{+} \mathrm{CD} 38^{+} \mathrm{T}$ cell singlets

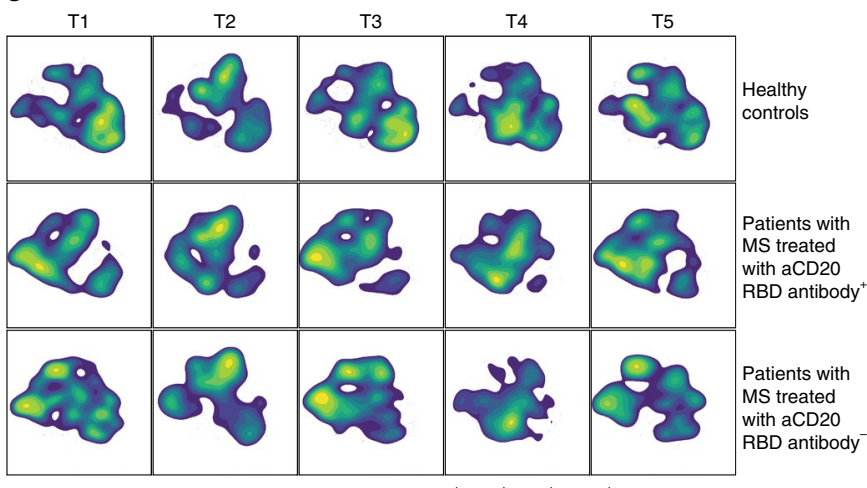

Gated on live $\mathrm{CD}^{+} \mathrm{CD} 8^{+} \mathrm{Ki} 67^{+} \mathrm{CD} 38^{+} \mathrm{T}$ cell singlets
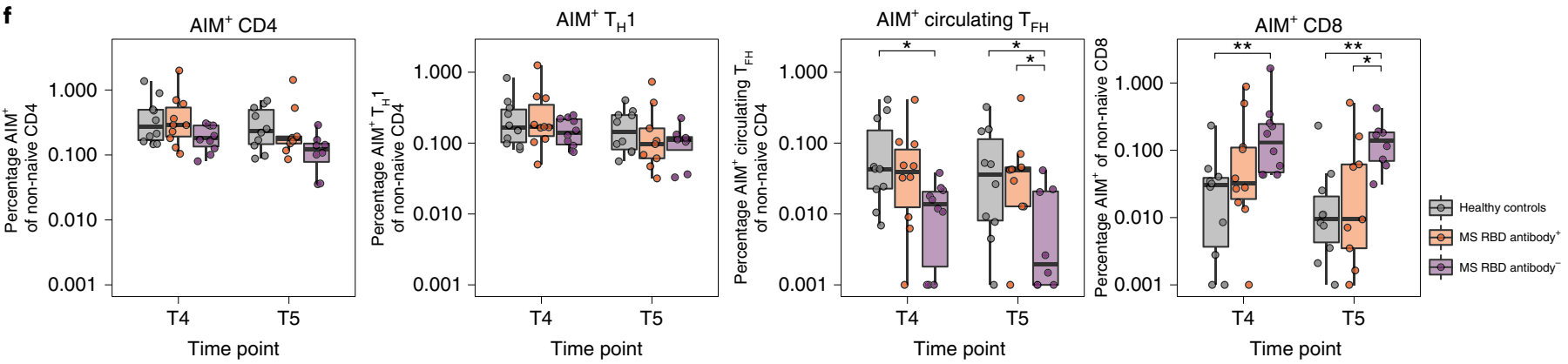

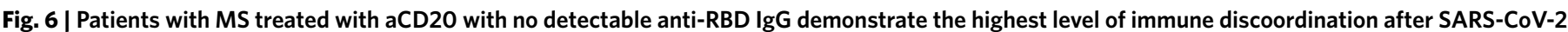
mRNA vaccination. a, Correlations of antigen-specific features at T2, T4 and T5 in healthy controls (left, $n=10$ ) and patients with MS treated with aCD20 (right, $n=20$ ). Associations were calculated using Spearman rank correlation; ${ }^{\star} P<0.05$. b,c, Correlations between the frequency of T4 AIM ${ }^{+}$circulating $\mathrm{T}_{\mathrm{FH}}$ cells and T4 anti-spike IgG (left) or spike-specific memory B cells (right) (b) and between the frequency of T5 AIM+CD8 T cells and T5 anti-spike IgG

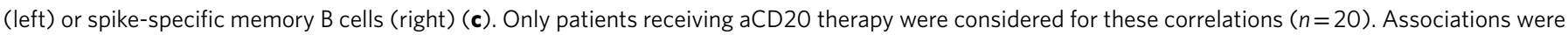
calculated using Spearman rank correlation and are shown with Pearson trend lines for visualization. d,e, Opt-SNE projections of concatenated cytometry data for activated $\mathrm{Ki} 67+\mathrm{CD} 38+\mathrm{CD} 4$ (d) and activated Ki67+CD38+ CD8 (e) T cells for each time point and group combination are shown. Groups: healthy

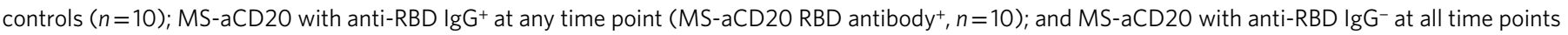
examined (MS-aCD20 RBD antibody $\left.{ }^{-}, n=10\right)$. f, AIM+ frequencies of the indicated T cell populations after mRNA vaccination at T4 and T5. The values represent the background-subtracted frequency of $\mathrm{AIM}^{+}$non-naive T cells above paired baseline frequencies for healthy controls ( $\mathrm{gray}, n=10$ ), $\mathrm{MS}$ RBD antibody ${ }^{+}$(orange, $n=10$ ) and MS RBD antibody ${ }^{-}$(purple, $n=10$ ) groups. Statistics were calculated using an unpaired, two-tailed Wilcoxon test. ${ }^{\star} P<0.05$, $\star \star P<0.01$.

some overlapping temporal features of change during the course of vaccination. By contrast, the RBD antibody ${ }^{-}$MS-aCD20 group displayed a distinct opt-SNE projection of $\mathrm{Ki} 67^{+} \mathrm{CD} 38^{+} \mathrm{CD} 4$ $\mathrm{T}$ cells with minimal vaccine-induced changes. To quantify these differences, we used the earth mover's distance (EMD) metric for all pair-wise comparisons to calculate similarities between probability distributions within the opt-SNE maps. EMD revealed similarity in the overall landscape of activated CD4 T cells between healthy controls and RBD antibody ${ }^{+}$MS-aCD20 patients, whereas the RBD antibody ${ }^{-}$MS-aCD20 group was highly dissimilar to the other groups at all time points (Extended Data Figure 10a). By contrast to activated CD4 T cells, vaccine-induced changes in the activated CD8 $\mathrm{T}$ cell compartment after the first dose (T2) were more similar in RBD antibody ${ }^{+}$and antibody ${ }^{-}$MS-aCD20 groups, both of which resembled the healthy control responses (Fig. 6e and Extended Data Figure 10b). However, after the second vaccine 
dose (T4), the RBD antibody ${ }^{+} \mathrm{MS}$-aCD20 group was different from both the healthy control and $\mathrm{RBD}^{-}$MS-aCD20 groups (Fig. 6e and Extended Data Figure 10b) due to the larger presence of metacluster 8. Taken together, these data show that, in the absence of a functional humoral response using anti-RBD IgG as a proxy, the defects identified in vaccine-induced responses of activated CD4 $\mathrm{T}$ cells in patients with MS treated with aCD20 were amplified. By contrast, vaccine-induced CD8 T cell responses were more similar in patients with MS treated with aCD20 compared to healthy controls with less impact of anti-RBD IgG status.

Finally, we assessed whether the differential vaccine responses of the CD4 and CD8 T cell subsets in patients with MS treated with aCD20 separated by RBD IgG response were related to the induction of antigen-specific T cell responses. The MS-aCD20 RBD antibody ${ }^{-}$group showed markedly lower abundance of $\mathrm{AIM}^{+}$circulating $\mathrm{T}_{\mathrm{FH}}$ cells at $\mathrm{T} 4$ and $\mathrm{T} 5$ (Fig. 6f). By contrast, $\mathrm{AIM}^{+} \mathrm{T}_{\mathrm{H}} 1$ cells were similar in RBD antibody ${ }^{+}$and $\mathrm{RBD}$ antibody ${ }^{-}$groups. Notably, $\mathrm{AIM}^{+} \mathrm{CD} 8 \mathrm{~T}$ cell vaccine responses were significantly more robust in MS-aCD20 RBD antibody ${ }^{-}$patients compared to RBD antibody ${ }^{+}$ patients after the second vaccine dose, supporting the notion that SARS-CoV-2 mRNA vaccine-induced CD8 T cell responses were more vigorous in patients who lacked $\mathrm{B}$ cells and antibody responses due to aCD20 treatment. Together, these data underscore the interrelated and coordinated nature of mRNA vaccine-induced immune responses and shed light on underlying 'immune health' differences in patients with MS on aCD20 therapy.

\section{Discussion}

The goal of this study was to evaluate the impact of aCD20 therapy on SARS-CoV-2 mRNA vaccine responses. Therapy with aCD20 is used in many clinical settings including cancer immunotherapy, rheumatology and neurology. In MS, aCD20 treatment is commonly used as monotherapy offering the advantage of studying its effects in a patient population relatively less confounded by other concurrent immune therapies.

Although neutralizing antibodies are likely to be important in vaccine-induced protection, precise correlates of immunity are incompletely defined and recent evidence also points to a role for $\mathrm{T}$ cells ${ }^{36-39,48}$. Despite poor antibody responses in most patients with MS treated with aCD20, all of these patients generated robust CD4 and CD8 $\mathrm{T}$ cell responses to SARS-CoV-2 mRNA vaccination suggesting that vaccinating $B$ cell-deficient patients is still likely to provide some measure of immunity to SARS-CoV-2, especially considering that $\mathrm{T}$ cells may retain recognition of emerging variants of concern that have escaped antibody neutralization $^{40,41}$. Despite this preserved $\mathrm{T}$ cell priming, patients with MS treated with aCD20 had selective defects in antigen-specific circulating $\mathrm{T}_{\mathrm{FH}}$ responses compared to healthy controls, an effect more severe in patients with MS treated with aCD20 who lacked RBD antibody responses. Although it is possible that some of these changes could reflect an impact of aCD20 on a subset of $\mathrm{CD} 20^{+} \mathrm{T}$ cells ${ }^{49,50}$, these data are also consistent with the idea that not only do $\mathrm{T}_{\mathrm{FH}}$ cells provide help to $\mathrm{B}$ cells ${ }^{51}$, but that germinal center $\mathrm{B}$ cells also influence optimal $\mathrm{T}_{\mathrm{FH}}$ cell responses ${ }^{52}$. By contrast, $\mathrm{T}_{\mathrm{H}} 1$ cell responses were only mildly impacted and CD8 $\mathrm{T}$ cell responses were augmented, especially after the second vaccine dose. Although these $\mathrm{T}$ cell responses are promising indicators of immunity in patients with MS treated with aCD20, future clinical studies examining the rate of SARS-CoV-2 infection, vulnerability to variants of concern and COVID-19 outcomes in patients with primary and secondary B cell immunodeficiencies will be necessary to fully interrogate the degree of clinical protection in these patients after mRNA vaccination.

$B$ cell reconstitution in the circulation was, as expected, preferentially detected in patients who were farther removed from their last aCD20 treatment. This patient subgroup more efficiently generated antibodies and memory B cells against spike and RBD and had less perturbed $\mathrm{CD} 4$ and $\mathrm{CD} 8 \mathrm{~T}$ cell responses to $\mathrm{mRNA}$ vaccination. The magnitude of vaccine-induced humoral responses correlated better with the extent of $B$ cell reconstitution at the time of vaccination than with the time window between vaccination and the last aCD20 infusion, suggesting that the underlying mechanism for this effect is $\mathrm{B}$ cell reconstitution. Thus, assessing reemergence of peripheral B cells may be a better marker than time since last aCD20 treatment to determine which patients will generate humoral immunity after vaccination and future booster doses.

One unexpected finding was the more robust and functionally competent vaccine-induced CD8 $\mathrm{T}$ cell response after SARS-CoV-2 mRNA vaccination in the patients with MS treated with aCD20, especially patients who failed to generate anti-RBD IgG. This difference was most prominent after the administration of the second vaccine dose. These results are evidence of effective immune priming by mRNA vaccines in the absence of circulating B cells, findings that may also be relevant for the application of mRNA vaccines in other settings, such as neoantigen cancer vaccines in patients with $\mathrm{B}$ cell deficiencies ${ }^{53}$. An important question to address in the future is the underlying mechanism of this augmented CD8 $\mathrm{T}$ cell response. One possibility is that, in the absence of antibody, there is an increased abundance of antigen to drive CD8 $\mathrm{T}$ cell activation and proliferation due to lack of antigen clearance by vaccine-induced antibodies. Alternatively, regulatory $\mathrm{B}$ cells may play a direct role in attenuating CD8 T cell responses ${ }^{54,55}$. A third possibility is through the effects of antibody or immune complexes via engagement of the inhibitory Fc receptor Fc $\gamma$ RIIB on dendritic cells ${ }^{56,57}$ or CD8 T cells ${ }^{58}$. Future studies will be necessary to determine the contribution of these possible mechanisms.

Overall, these studies provide strong evidence of immune priming by SARS-CoV-2 mRNA vaccines in patients with MS treated with aCD20. Although most of these patients do not generate optimal antibody responses, $\mathrm{T}$ cell priming, especially of $\mathrm{T}_{\mathrm{H}} 1$ and $\mathrm{CD} 8 \mathrm{~T}$ cells, is largely intact. However, treatment with aCD20 and B cell deficiency were associated with altered coordination of the immune response and circulating $\mathrm{T}_{\mathrm{FH}}$ responses were compromised. Nevertheless, despite the intent of aCD20 treatment to remove $\mathrm{B}$ cell-mediated immunity, including the effects of B cells in presenting antigen to CD4 T cells, these studies reveal variable levels of residual underlying immune functionality in patients with MS treated with aCD20. It will be important in the future to determine whether the residual humoral immunity and sustained or augmented $\mathrm{T}_{\mathrm{H}} 1$ or $\mathrm{CD} 8 \mathrm{~T}$ cell responses, respectively, retain the ability to respond to emerging variants of concern of SARS-CoV-2. We also note that analysis of mRNA vaccine-induced immune responses serves not only to measure immunity to SARS-CoV-2 but also as an 'analytical vaccine' offering insights into the underlying immune health and fitness of patients with MS treated with aCD20. Overall, these data provide key insights about the ability to generate immune responses in immunocompromised populations that will be relevant for clinical guidance in these patients and possible public health recommendations for vulnerable populations.

\section{Online content}

Any methods, additional references, Nature Research reporting summaries, source data, extended data, supplementary information, acknowledgements, peer review information; details of author contributions and competing interests; and statements of data and code availability are available at https://doi.org/10.1038/ s41591-021-01507-2.

Received: 22 June 2021; Accepted: 16 August 2021;

Published online: 14 September 2021 


\section{References}

1. Yang, J. et al. Disease burden and clinical severity of the first pandemic wave of COVID-19 in Wuhan, China. Nat. Commun. 11, 5411 (2020).

2. Miller, I. F., Becker, A. D., Grenfell, B. T. \& Metcalf, C. J. E. Disease and healthcare burden of COVID-19 in the United States. Nat. Med. 26, 1212-1217 (2020)

3. Kyriakidis, N. C., López-Cortés, A., González, E. V., Grimaldos, A. B. \& Prado, E. O. SARS-CoV-2 vaccines strategies: a comprehensive review of phase 3 candidates. NPJ Vaccines 6, 28 (2021).

4. Polack, F. P. et al. Safety and efficacy of the BNT162b2 mRNA Covid-19 vaccine. N. Engl. J. Med. 383, 2603-2615 (2020).

5. Baden, L. R. et al. Efficacy and safety of the mRNA-1273 SARS-CoV-2 vaccine. N. Engl. J. Med. 384, 403-416 (2021)

6. Sormani, M. P. et al. Disease-modifying therapies and coronavirus disease 2019 severity in multiple sclerosis. Ann. Neurol. 89, 780-789 (2021).

7. Duléry, R. et al. Prolonged in-hospital stay and higher mortality after Covid-19 among patients with non-Hodgkin lymphoma treated with B-cell depleting immunotherapy. Am. J. Hematol. 96, 934-944 (2021).

8. Avouac, J. et al. COVID-19 outcomes in patients with inflammatory rheumatic and musculoskeletal diseases treated with rituximab: a cohort study. Lancet Rheumatol. 3, e419-e426 (2021).

9. Strangfeld, A. et al. Factors associated with COVID-19-related death in people with rheumatic diseases: results from the COVID-19 Global Rheumatology Alliance physician-reported registry. Ann. Rheum. Dis. 80, 930-942 (2021).

10. Louapre, C. et al. Clinical characteristics and outcomes in patients with coronavirus disease 2019 and multiple sclerosis. JAMA Neurol. 77, 1079-1088 (2020)

11. Loonstra, F. C., Hoitsma, E., van Kempen, Z. L. E., Killestein, J. \& Mostert, J. P. COVID-19 in multiple sclerosis: the Dutch experience. Mult. Scler. 26, 1256-1260 (2020)

12. Zabalza, A. et al. COVID-19 in multiple sclerosis patients: susceptibility, severity risk factors and serological response. Eur. J. Neurol.10.1111/ene.14690 (2020).

13. Shanehbandi, D., Majidi, J., Kazemi, T., Baradaran, B. \& Aghebati-Maleki, L. CD20-based immunotherapy of B-cell derived hematologic malignancies. Curr. Cancer Drug Targets 17, 423-444 (2017).

14. Lee, D. S. W., Rojas, O. L. \& Gommerman, J. L. B cell depletion therapies in autoimmune disease: advances and mechanistic insights. Nat. Rev. Drug Discov. 20, 179-199 (2021)

15. McGinley, M. P., Goldschmidt, C. H. \& Rae-Grant, A. D. Diagnosis and treatment of multiple sclerosis: a review. JAMA 325, 765-779 (2021).

16. Comi, G. et al. Role of B cells in multiple sclerosis and related disorders. Ann. Neurol. 89, 13-23 (2021).

17. Nutt, S. L., Hodgkin, P. D., Tarlinton, D. M. \& Corcoran, L. M. The generation of antibody-secreting plasma cells. Nat. Rev. Immunol. 15, 160-171 (2015).

18. Bar-Or, A. et al. Effect of ocrelizumab on vaccine responses in patients with multiple sclerosis: the VELOCE study. Neurology 95, e1999-e2008 (2020)

19. Loebermann, M. et al. Vaccination against infection in patients with multiple sclerosis. Nat. Rev. Neurol. 8, 143-151 (2012).

20. Olberg, H. K. et al. Immunotherapies influence the influenza vaccination response in multiple sclerosis patients: an explorative study. Mult. Scler. 20, 1074-1080 (2014)

21. Eisenberg, R. A. et al. Rituximab-treated patients have a poor response to influenza vaccination. J. Clin. Immunol. 33, 388-396 (2013).

22. van Assen, S. et al. Humoral responses after influenza vaccination are severely reduced in patients with rheumatoid arthritis treated with rituximab. Arthritis Rheum. 62, 75-81 (2010).

23. Bingham, C. O.3rd et al. Immunization responses in rheumatoid arthritis patients treated with rituximab: results from a controlled clinical trial. Arthritis Rheum. 62, 64-74 (2010).

24. Deepak, P. et al. Glucocorticoids and B cell depleting agents substantially impair immunogenicity of mRNA vaccines to SARS-CoV-2. Preprint at medRxiv https://doi.org/10.1101/2021.04.05.21254656 (2021).

25. Achiron, A. et al. Humoral immune response to COVID-19 mRNA vaccine in patients with multiple sclerosis treated with high-efficacy disease-modifying therapies. Ther. Adv. Neurol. Disord. 14, 17562864211012836 (2021)

26. Konchese, F. \& Hausmann, B. B lymphocytes in vivo fail to prime naive T cells but can stimulate antigen-experienced T lymphocytes. J. Exp. Med. 177, 679-690 (1993)

27. Sunshine, G. H., Jimmo, B. L., Ianelli, C. \& Jarvis, L. Strong priming of T cells adoptively transferred into scid mice. J. Exp. Med. 174, 1653-1656 (1991).

28. Asano, M. S. \& Ahmed, R. CD8 T cell memory in B cell-deficient mice. J. Exp. Med. 183, 2165-2174 (1996).

29. Liu, Y. et al. Gene-targeted B-deficient mice reveal a critical role for B cells in the CD4 T cell response. Int. Immunol. 7, 1353-1362 (1995).
30. Ron, Y., De Baetselier, P., Gordon, J., Feldman, M. \& Segal, S. Defective induction of antigen-reactive proliferating $\mathrm{T}$ cells in B cell-deprived mice. Eur. J. Immunol. 11, 964-968 (1981).

31. Chan, O. T., Hannum, L. G., Haberman, A. M., Madaio, M. P. \& Shlomchik, M. J. A novel mouse with B cells but lacking serum antibody reveals an antibody-independent role for B cells in murine lupus. J. Exp. Med. 189, 1639-1648 (1999)

32. Barnett, L. G. et al. B cell antigen presentation in the initiation of follicular helper T cell and germinal center differentiation. J. Immunol. 192, 3607-3617 (2014).

33. Kurt-Jones, E. A. et al. The role of antigen-presenting B cells in T cell priming in vivo. Studies of B cell-deficient mice.J. Immunol. 140, 3773-3778 (1988)

34. Crawford, A., MacLeod, M., Schumacher, T., Corlett, L. \& Gray, D. Primary $\mathrm{T}$ cell expansion and differentiation in vivo requires antigen presentation by B cells. J. Immunol. 176, 3498-3506 (2006).

35. Giles, J. R., Kashgarian, M., Koni, P. A. \& Shlomchik, M. J. B cell-specific MHC class II deletion reveals multiple nonredundant roles for B cell antigen presentation in murine lupus. J. Immunol. 195, 2571-2579 (2015).

36. Dan, J. M. et al. Immunological memory to SARS-CoV-2 assessed for up to 8 months after infection. Science 371, eabf4063 (2021)

37. Sekine, T. et al. Robust $\mathrm{T}$ cell immunity in convalescent individuals with asymptomatic or mild COVID-19. Cell 183, 158-168.e14 (2020).

38. Rydyznski Moderbacher, C. et al. Antigen-specific adaptive immunity to SARS-CoV-2 in acute COVID-19 and associations with age and disease severity. Cell 183, 996-1012.e19 (2020).

39. Bange, E. M. CD8 ${ }^{+}$T cells contribute to survival in patients with COVID-19 and hematologic cancer.Nat. Med. 27, 1280-1289 (2021).

40. Tarke, A. et al. Impact of SARS-CoV-2 variants on the total $\mathrm{CD}^{+}$and $\mathrm{CD}^{+}$ $\mathrm{T}$ cell reactivity in infected or vaccinated individuals. Cell Rep. Med. 2, 100355 (2021).

41. Geers, D. et al. SARS-CoV-2 variants of concern partially escape humoral but not T-cell responses in COVID-19 convalescent donors and vaccinees.Sci. Immunol. 6, eabj1750 (2021)

42. Goel, R. R. Distinct antibody and memory B cell responses in SARS-CoV-2 naïve and recovered individuals following mRNA vaccination.Sci. Immunol. 6 , eabi6950 (2021)

43. Samanovic, M. I. et al. Robust immune responses after one dose of BNT162b2 mRNA vaccine dose in SARS-CoV-2 experienced individuals. Preprint at medRxivhttps://doi.org/10.1101/2021.02.07.21251311(2021).

44. Miller, J. D. et al. Human effector and memory CD8 ${ }^{+} \mathrm{T}$ cell responses to smallpox and yellow fever vaccines. Immunity 28, 710-722 (2008).

45. Herati, R. S. et al. Circulating CXCR $5^{+} \mathrm{PD}-1^{+}$response predicts influenza vaccine antibody responses in young adults but not elderly adults. J. Immunol. 193, 3528-3537 (2014).

46. Herati, R. S. et al. Successive annual influenza vaccination induces a recurrent oligoclonotypic memory response in circulating $\mathrm{T}$ follicular helper cells. Sci. Immunol. 2, eaag2152 (2017).

47. Painter, M. M. et al. Rapid induction of antigen-specific $\mathrm{CD}^{+} \mathrm{T}$ cells guides coordinated humoral and cellular immune responses to SARS-CoV-2 mRNA vaccination. Preprint at bioRxiv https://doi.org/10.1101/2021.04.21.440862 (2021).

48. Mathew, D. et al. Deep immune profiling of COVID-19 patients reveals distinct immunotypes with therapeutic implications. Science 369, eabc8511 (2020).

49. Palanichamy, A. et al. Rituximab efficiently depletes increased CD20-expressing T cells in multiple sclerosis patients. J. Immunol. 193, 580-586 (2014).

50. Schuh, E. et al. Features of human $\mathrm{CD}^{+} \mathrm{CD} 20^{+} \mathrm{T}$ cells. J. Immunol. 197, 1111-1117 (2016).

51. Crotty, S. T follicular helper cell biology: a decade of discovery and diseases. Immunity 50, 1132-1148 (2019).

52. Merkenschlager, J. et al. Dynamic regulation of $\mathrm{T}_{\mathrm{FH}}$ selection during the germinal centre reaction. Nature 591, 458-463 (2021).

53. Blass, E. \& Ott, P. A. Advances in the development of personalized neoantigen-based therapeutic cancer vaccines. Nat. Rev. Clin. Oncol. 18 215-229 (2021)

54. Mohib, K. et al. Antigen-dependent interactions between regulatory B cells and $\mathrm{T}$ cells at the T:B border inhibit subsequent $\mathrm{T}$ cell interactions with DCs. Am. J. Transplant. 20, 52-63 (2020).

55. Boldison, J., Da Rosa, L. C., Davies, J., Wen, L. \& Wong, F. S. Dendritic cells license regulatory B cells to produce IL-10 and mediate suppression of antigen-specific CD8 T cells. Cell. Mol. Immunol. 17, 843-855 (2020).

56. Li, F., Smith, P. \& Ravetch, J. V. Inhibitory Fcy receptor is required for the maintenance of tolerance through distinct mechanisms. J. Immunol. 192, 3021-3028 (2014)

57. Smith, K. G. C. \& Clatworthy, M. R. Fc $\gamma$ RIIB in autoimmunity and infection: evolutionary and therapeutic implications. Nat. Rev. Immunol. 10 328-343 (2010). 
58. Morris, A. B. et al. Signaling through the inhibitory Fc receptor FcyRIIB induces $\mathrm{CD} 8^{+} \mathrm{T}$ cell apoptosis to limit $\mathrm{T}$ cell immunity. Immunity $\mathbf{5 2}$, 136-150.e6 (2020).

Publisher's note Springer Nature remains neutral with regard to jurisdictional claims in published maps and institutional affiliations.

(c) (i) Open Access This article is licensed under a Creative Commons

Attribution 4.0 International License, which permits use, sharing, adaptation, distribution and reproduction in any medium or format, as long as you give appropriate credit to the original author(s) and the source, provide a link to the Creative Commons license, and indicate if changes were made. The images or other third party material in this article are included in the article's Creative Commons license, unless indicated otherwise in a credit line to the material. If material is not included in the article's Creative Commons license and your intended use is not permitted by statutory regulation or exceeds the permitted use, you will need to obtain permission directly from the copyright holder. To view a copy of this license, visit http://creativecommons. org/licenses/by/4.0/.

(๑) The Author(s) 2021 


\section{Methods}

Study design. In this longitudinal study, healthy controls $(n=10)$ and patients with MS treated with anti-CD20 $(n=20,19$ patients on ocrelizumab and 1 patient on rituximab) were recruited between December 2020 and April 2021. Plasma and PBMCs were collected immediately before the first vaccine dose (T1), 10-12d after the first vaccine dose (T2), immediately before the second vaccine dose (T3), $10-12 \mathrm{~d}$ after the second vaccine dose (T4) and $25-30 \mathrm{~d}$ after the second vaccine dose (T5). Clinical information for healthy controls and patients with MS treated with anti-CD20 vaccinees can be found in Extended Data Fig. 1. All experiments were conducted in blinded fashion with designated members of the clinical team (who were not part of running the assays) having access to the sample key until data were collected, at which point all researchers were unblinded for the analysis. All individuals enrolled in this study provided informed written consent as part of protocols approved by the University of Pennsylvania institutional review boards and in compliance with the October 2013 Declaration of Helsinki principles. Enrolled individuals did not receive compensation for their participation in the study.

Cell isolation and cryopreservation. Venous blood was collected in multiple 10-ml K2EDTA tubes (BD Vacutainer, catalog no. 366643; Becton, Dickinson and Company). Blood was diluted at a 1:1 ratio with PBS that contained $2 \mathrm{mM}$ of EDTA and then slowly transferred to a 50-ml tube that contained $15 \mathrm{ml} \mathrm{Ficoll} \mathrm{(catalog}$ no. CA95038-168L; GE Healthcare). Tubes were then spun at $700 \mathrm{~g}$ at room temperature with no brake. PBMC layers were collected using a transfer pipet and then washed once with $40 \mathrm{ml}$ of PBS +EDTA buffer before being submitted for cell counting. Cells were then resuspended in freezer media (human $\mathrm{AB}$ serum $+10 \%$ DMSO) and aliquoted into cryopreserved tubes (approximately 20 million per tube). PBMC samples were first stored in Mr. Frosty freezing containers at $-80^{\circ} \mathrm{C}$ and then transferred to liquid nitrogen tanks for long-term storage.

Plasma isolation. Venous blood was collected in a 10-ml K2EDTA tube. The tube was then stored upright at room temperature for $30 \mathrm{~min}$ before centrifugation at $4^{\circ} \mathrm{C}$ for 10 minutes at 2,500 $\mathrm{g}$ (with swinging bucket rotor). Supernatants were then collected, aliquoted and stored at $-80^{\circ} \mathrm{C}$ until further use

Detection of SARS-CoV-2-specific antibodies. Plasma samples were tested for SARS-CoV-2-specific antibody by ELISA ${ }^{59}$. The estimated sensitivity of the test is $100 \%$ ( $95 \%$ confidence interval (CI), 89.1 to $100.0 \%$ ) and specificity is $98.9 \%$ ( $95 \%$ CI, 98.0 to $99.5 \%$ ). Plasmids encoding the recombinant full-length spike protein and the RBD were provided by F. Krammer and purified by nickel-nitrilotriacetic acid resin (QIAGEN). Monoclonal antibody CR3022 was included on each plate to convert optical density values into relative antibody concentrations. Plasmids needed to express CR3022 were provided by I. Wilson.

SARS-CoV-2 neutralization assay. $293 \mathrm{~T}$ cells were transfected with pCG1 SARS-CoV-2 S D614G delta 18 expression plasmid encoding a codon-optimized SARS-CoV-2 S gene with an 18 residue truncation in the cytoplasmic tail (provided by S. Pohlmann). Twenty-four hours after transfection, the SARS-CoV-2 spike-expressing cells were infected for $2 \mathrm{~h}$ with vesicular stomatitis virus $\mathrm{G}$ pseudotyped VSV $\Delta$ G-red fluorescent protein (RFP) at a multiplicity of infection of approximately 1 . Media containing the VSV $\Delta$ G-RFP SARS-CoV-2 pseudotypes were collected $28-30 \mathrm{~h}$ after infection and clarified by centrifugation. For the antibody neutralization assay using VSV $\Delta$ G-RFP SARS-CoV-2, all sera were heat-inactivated for $30 \mathrm{~min}$ at $55^{\circ} \mathrm{C}$ before use in the neutralization assay. Vero E6 cells stably expressing transmembrane protease serine 2 were seeded in a 96-well collagen-coated plate; the next day, twofold serially diluted serum samples were mixed with VSV $\Delta$ G-RFP SARS-CoV-2 pseudotype virus (100-300 focus-forming units per well) and incubated for $1 \mathrm{~h}$ at $37^{\circ} \mathrm{C}$. Also included in this mixture to neutralize any potential carryover vesicular stomatitis virus $\mathrm{G}$ was $1 \mathrm{E} 9 \mathrm{~F}$, a mouse anti-VSV Indiana G, at a concentration of $600 \mathrm{ng} \mathrm{ml}^{-1}$ (Ab01402-2.0; Absolute Antibody). The serum-virus mixture was then used to replace the media on the Vero E6 transmembrane protease serine 2 cells. Twenty-two hours after infection, cells were washed and fixed with $4 \%$ paraformaldehyde before visualization on an S6 FluoroSpot Analyzer (CTL). Individual infected foci were enumerated and the values compared with control wells without antibody. The focus reduction neutralization titer $50 \%\left(\mathrm{FRNT}_{50}\right)$ was measured as the greatest serum dilution at which the focus count was reduced by at least 50\% relative to control cells that were infected with pseudotype virus in the absence of human serum. Focus reduction neutralization titers $50 \%$ for each sample were measured in at least 2 technical replicates and were reported for each sample as the geometric mean of the technical replicates.

Flow cytometry. Samples were acquired on a 5 laser BD FACS Symphony A5 (X50 SORP). Standardized SPHERO rainbow beads (catalog no. RFP-30-5A; Spherotech) were used to track and adjust photomultiplier tubes over time. UltraComp eBeads (catalog no. 01-2222-42; Thermo Fisher Scientific) were used for compensation. Up to $1 \times 10^{6}$ PBMCs were acquired for each sample. All antibodies used for high-dimensional FACS analysis can be found in Supplementary Table 2. All data collection was done using the BD FACSDiva Software (version 9.0).
Detection of SARS-CoV-2-specific memory B cells. Antigen-specific B cells were detected using biotinylated proteins in combination with different streptavidin-fluorophore conjugates ${ }^{42}$. Biotinylated proteins were multimerized with fluorescently labeled streptavidin for $1 \mathrm{~h}$ at $4^{\circ} \mathrm{C}$. Full-length spike protein (R\&D Systems) was mixed with streptavidin-Brilliant Violet 421 (BioLegend) at a 10:1 mass ratio (for example, $200 \mathrm{ng}$ spike with $20 \mathrm{ng}$ streptavidin; approximately 4:1 molar ratio). Spike RBD (R\&D Systems) was mixed with streptavidin allophycocyanin (BioLegend) at a 2:1 mass ratio (for example, $25 \mathrm{ng}$ RBD with $12.5 \mathrm{ng}$ streptavidin; approximately 4:1 molar ratio). Biotinylated influenza hemagglutinin pools (A/Brisbane/02/2018/H1N1, B/Colorado/06/2017; Immune Technology) were mixed with streptavidin-phycoerythrin (BioLegend) at a $6.25: 1$ mass ratio (for example, $100 \mathrm{ng}$ hemagglutinin pool with $16 \mathrm{ng}$ streptavidin; approximately 6:1 molar ratio). Streptavidin-Brilliant Violet 711 (BD Biosciences) was used as a decoy probe without biotinylated protein to gate out cells that nonspecifically bind streptavidin. Antigen probes for spike, $\mathrm{RBD}$ and hemagglutinin were prepared individually and mixed together after multimerization with $5 \mu \mathrm{M}$ of free D-biotin (Avidity LLC) to minimize potential cross-reactivity between probes.

\section{AIM assays. PBMCs were thawed by diluting with $10 \mathrm{ml}$ of warm RPMI} supplemented with $10 \% \mathrm{FCS}, 2 \mathrm{mM}$ of L-glutamine, $100 \mathrm{U} \mathrm{ml}^{-1}$ of penicillin and $100 \mathrm{mg} \mathrm{ml}^{-1}$ streptomycin (R10) and washed once in R10. Cell counts were obtained with a Countess Automated Cell Counter (Thermo Fisher Scientific) and each sample was resuspended in fresh R10 to a density of $5 \times 10^{6} \mathrm{cells} \mathrm{per} \mathrm{ml}^{-1}$. For each condition, duplicate wells containing $1 \times 10^{6}$ cells in $200 \mathrm{ml}$ were plated in 96-well round-bottom plates and rested overnight in a humidified incubator at $37^{\circ} \mathrm{C}$ and $5 \% \mathrm{CO}_{2}$. After resting, $\mathrm{CD} 40$-blocking antibody $\left(0.5 \mathrm{mg} \mathrm{ml}^{-1}\right.$ final concentration) was added to the cultures for $15 \mathrm{~min}$ before stimulation and cells were subsequently stimulated for $24 \mathrm{~h}$ with costimulation (antihuman CD28/ CD49d; BD Biosciences) and peptide megapools (CD4-S for all CD4 T cell analyses, CD8-E for all CD8 T cell analyses) at a final concentration of $1 \mathrm{mg} \mathrm{ml}^{-1}$ (refs. ${ }^{60,61}$ ). Matched unstimulated samples for each donor at each time point were treated with costimulation alone; $20 \mathrm{~h}$ poststimulation, antibodies targeting CXCR3 (clone G02587, dilution 1:800, catalog no. 353716; BioLegend), CCR7 (clone G043H7, dilution 1:400, catalog no. 353234; BioLegend), CD40L (clone 2431, dilution 1:50, catalog no. 310838; BioLegend), CXCR5 (clone RF8B2, dilution 1:100, catalog no. 565191; BD Biosciences) and CCR6 (clone G034E3, dilution $1: 800$, catalog no. 353432; BioLegend) were added to the culture along with monensin (GolgiStop; BD Biosciences) for a 4 -h stain at $37^{\circ} \mathrm{C}$. After $4 \mathrm{~h}$, duplicate wells were pooled and cells were washed in PBS supplemented with 2\% FCS (FACS buffer). Cells were stained for $10 \mathrm{~min}$ at room temperature with Ghost Dye Violet 510 and Fc receptor blocking solution (Human TruStain FcX; BioLegend) and washed once in FACS buffer. Surface staining for $30 \mathrm{~min}$ at room temperature was then performed with antibodies directed against: CD4 (clone SK3, dilution 1:400, catalog no. 563550; BD Biosciences); CD8 (clone RPA-T8, dilution 1:400, catalog no. 612943; BD Biosciences); CD45RA (clone HI100, dilution 1:2,000, catalog no. 751555; BD Biosciences); CD27 (clone L128, dilution 1:400, catalog no. 612829; BD Biosciences); CD3 (clone UCHT1, dilution 1:800, catalog no. 612896; BD Biosciences); CD40L (clone 24-31, dilution 1:50, catalog no. 310838; BioLegend); CD200 (clone A18042B, dilution 1:100, catalog no. 399804; BioLegend); OX40 (clone Ber-ACT35, dilution 1:1,600, catalog no. 350012; BioLegend); CD69 (clone FN50, dilution 1:400, catalog no. 310938; BioLegend); CD107a (clone H4A3, catalog no. 328644, dilution 1:100; BioLegend); granzyme B (clone GB11, catalog no. GRB17, dilution 1:3,200; Thermo Fisher Scientific); and 41BB (clone 4B4-1, dilution 1:400, catalog no. 309810; BioLegend) in FACS buffer. Cells were washed once in FACS buffer, fixed and permeabilized for $30 \mathrm{~min}$ at room temperature (Foxp3/Transcription Factor Fixation/Permeabilization Concentrate and Diluent; Invitrogen) and washed once in $1 \times$ permeabilization buffer before staining for intracellular IFN $\gamma$ (clone 4S.B3, dilution 1:400, catalog no. 502515; BioLegend), TNF (clone MAb11, dilution 1:800, catalog no. 12-7349-82; Thermo Fisher Scientific) and IL-2 (clone MQ1-17H12, dilution 1:500, catalog no. 500328; BioLegend) overnight at $4{ }^{\circ} \mathrm{C}$. Cells were then washed once and resuspended in $1 \%$ paraformaldehyde in PBS before data acquisition.

All data from the AIM expression assays were background-subtracted using paired unstimulated control samples. For memory $\mathrm{T}$ cell and helper $\mathrm{T}$ cell subsets, the $\mathrm{AIM}^{+}$background frequency of non-naive $\mathrm{T}$ cells was subtracted independently for each subset. $\mathrm{AIM}^{+}$cells were identified from non-naive $\mathrm{T}$ cell populations. $\mathrm{AIM}^{+} \mathrm{CD} 4 \mathrm{~T}$ cells were defined by dual expression of CD200 and CD40L. AIM ${ }^{+} \mathrm{CD} 8 \mathrm{~T}$ cells were defined by dual expression of $41 \mathrm{BB}$ and intracellular IFN $\gamma$.

High-dimensional data analysis of flow cytometry data. Opt-SNE ${ }^{62}$ and FlowSOM $^{63}$ analyses were performed using OMIQ (https://app.omiq.ai/). Total CD4, activated CD4 and activated CD8 T cells were analyzed separately. Markers used for all three analyses were CD27, CD45RA, CD127, T-bet, CXCR5, CD71, CD38, CCR6, HLA-DR, CTLA-4, PD-1, CCR7, CD25, CXCR3, ICOS, CXCR4, FOXP3 and Ki67. The opt-SNE parameters were: total CD4 T cells: maximum iterations 1,000 , perplexity 30 , theta 0.5 , seed 1234 , subsampling equal between cohorts: total $3 \mathrm{M}$ cells $(1.5 \mathrm{M}$ for healthy controls and $1.5 \mathrm{M}$ for patients with 
MS treated with aCD20 groups); activated $\mathrm{Ki} 67^{+} \mathrm{CD} 38^{+} \mathrm{CD} 4 \mathrm{~T}$ cells: maximum iterations 1,000 , perplexity 30 , theta 0.5 , components 2 , seed 1234 , subsampling equal between cohorts: total 13,822 cells $(6,911$ cells for healthy controls and 6,911 cells for patients with MS treated with aCD20); activated $\mathrm{Ki} 67^{+} \mathrm{CD} 38^{+} \mathrm{CD} 8 \mathrm{~T}$ cells: maximum iterations 1,000 , perplexity 30 , theta 0.5 , components 2 , seed 1234 , subsampling equal between cohorts: total 54,446 cells $(27,223$ cells for healthy controls and 27,223 cells for patients with MS treated with aCD20).

FlowSOM was performed in all three analyses using the same markers outlined above for opt-SNE and with the following parameters: number of clusters 100; number of metaclusters 10 (activated CD4, activated CD8 T cells) or 15 (total CD4 T cells); distance metric Euclidean; and consensus metaclustering.

To group individual samples on the basis of their $\mathrm{T}$ cell landscape, pair-wise EMD values were calculated on the opt-SNE axes for all healthy controls and patients with MS treated with aCD20 vaccinees at all time points collected using the emdist package v.0.3-1 in R v.4.0.5 $5^{6,65}$.

Statistical analysis. Owing to the heterogeneity of clinical and flow cytometry data, nonparametric tests of association were preferentially used throughout this study unless otherwise specified. Correlation coefficients between ordered features (including discrete ordinal, continuous scale or a mixture of the two) were quantified by the Spearman rank correlation coefficient; significance was assessed by the corresponding nonparametric methods (null hypothesis: $\rho=0$ ). Tests of association between mixed continuous versus nonordered categorical variables were performed by unpaired Wilcoxon test (for $n=2$ categories). The association between categorical variables was assessed by Fisher's exact test. All tests were performed in a two-sided manner, using a nominal significance threshold of $P<0.05$ unless otherwise specified. Other details, if any, for each experiment are provided in the relevant figure legends. Data analysis was done with the following software: R v.4.0.5; RStudio v.1.4.1106; emdist v.0.3-1; OMIQ release 2021; and Prism v.9.1.2 (GraphPad Software). ${ }^{*} P<0.05,{ }^{* *} P<0.01,{ }^{* * *} P<0.001$, ${ }^{* * * * P}<0.0001$.

Reporting Summary. Further information on research design is available in the Nature Research Reporting Summary linked to this article.

\section{Data availability}

Data are available in the main text, figures, extended data figures and supplementary materials. Raw FCS files can be accessed through the following links. Flow cytometry files for B cell analysis (Fig. 1, Extended Data Fig. 3): https://premium.cytobank.org/cytobank/experiments/378970; flow cytometry files for the high-dimensional analysis (Figs. 2, 4, 6 and Extended Data Figs. 4, 5, 7, 10 and Supplementary Fig. 1): https://premium.cytobank.org/cytobank/ experiments/378712; flow cytometry files for the AIM T cell analysis (Figs. 3, 5, 6 and Extended Data Figs. 6 and 8): https://premium.cytobank.org/cytobank/ experiments/378713. Datasets on Cytobank can be accessed via a registered account, which can be obtained by visiting the website https://www.cytobank.org. The key linking the participant IDs with the FCS filenames above is provided as a CSV file in the supplementary information. The serological information of the study participants is provided as a CSV file in the supplementary information. For any additional information on the participants, please email the corresponding author A. Bar-Or (with proper institutional review board approval, when applicable, from the requesting party) at amitbar@pennmedicine.upenn.edu.

\section{References}

59. Flannery, D. D. et al. SARS-CoV-2 seroprevalence among parturient women in Philadelphia. Sci. Immunol. 5, eabd5709 (2020).

60. Grifoni, A. et al. Targets of T cell responses to SARS-CoV-2 coronavirus in humans with COVID-19 disease and unexposed individuals. Cell 181, 1489-1501.e15 (2020).

61. Tarke, A. et al. Comprehensive analysis of $\mathrm{T}$ cell immunodominance and immunoprevalence of SARS-CoV-2 epitopes in COVID-19 cases. Cell Rep. Med. 2, 100204 (2021).

62. Belkina, A. C. et al. Automated optimized parameters for T-distributed stochastic neighbor embedding improve visualization and analysis of large datasets. Nat. Commun. 10, 5415 (2019).

63. Van Gassen, S. et al. FlowSOM: using self-organizing maps for visualization and interpretation of cytometry data. Cytometry A 87, 636-645 (2015).

64. Orlova, D. Y. et al. Earth Mover's Distance (EMD): a true metric for comparing biomarker expression levels in cell populations. PLOS ONE 11, e0151859 (2016).
65. Greenplate, A. R. et al. Computational immune monitoring reveals abnormal double-negative $\mathrm{T}$ cells present across human tumor types. Cancer Immunol. Res. 7, 86-99 (2019).

\section{Acknowledgements}

We thank the study participants for their generosity in making the study possible. We also thank the members of the Wherry and Bar-Or labs for helpful discussions and feedback. This work was supported by grants from the National Institutes of Health (NIH) nos. AI105343, AI082630, AI108545, AI155577, AI149680 (to E.J.W.), AI152236 (to P.B.), P30-AI0450080 (to E.T.L.P.), R01 AI118694 and UC4 DK112217 (to M.R.B.), T32 AR076951-01 (to S.A.A.), T32 CA009140 (to D.M.), U19AI082630 (to S.E.H. and E.J.W.), UM1 AI144288 (A.B.O.), NMSS SI-2011-37160 (A.B.O.), funding from the Allen Institute for Immunology (to S.A.A., E.J.W.), Chen Family Research Fund (to S.A.A.), the National Multiple Sclerosis Society-American Brain Foundation Clinician Scientist Award (to M.K.), Parker Institute for Cancer Immunotherapy (to E.J.W.), Penn Center for Research on Coronavirus and Other Emerging Pathogens (to P.B.), University of Pennsylvania Perelman School of Medicine COVID Fund (to R.R.G., E.J.W.), University of Pennsylvania Institute for Immunology Glick COVID-19 research award (to M.R.B.), University of Pennsylvania Perelman School of Medicine 21st Century Scholar Fund (to R.R.G.) and a philanthropic gift from J. Lurie, J. Embiid, J. Harris and D. Blitzer (to S.E.H.). Work in the Wherry lab is supported by the Parker Institute for Cancer Immunotherapy. This work was also supported by NIH contract no. $75 \mathrm{~N} 9301900065$ (to D.W. and A.S.)

\section{Author contributions}

E.J.W., R.L. and A.B.O. conceived the study. S.A.A., M.M.P., R.R.G., R.L., D.M., S.G. and K.A.L. carried out the experiments. M.K., D.J., C.E.M., R.L. and A.B.O. were involved in clinical recruitment. R.L., A.R. and J.C.K. processed the peripheral blood samples and managed the sample database. D.W., A.G. and A.S. provided the data and materials. A.R.G., E.J.W., R.L. and A.B.O. supervised the study. S.A.A., M.K., M.M.P., R.R.G., D.M., K.L., A.R., K.R.P., D.A.E., J.C.K., D.M.M., C.E.M., I.M., D.J., A.B., M.R.B., E.T.L.P., D.W., A.G., K.A.L., S.G., A.S., P.B., S.E.H., A.R.G., E.J.W., R.L. and A.B.O. participated in data analysis, interpretation and manuscript review. S.A.A., E.J.W., R.L. and A.B.O. wrote the manuscript.

\section{Competing interests}

S.E.H. has received consultancy fees from Sanofi-Pasteur, Lumen, Novavax and Merk for work unrelated to this article. E.J.W. is consulting or is an advisor for Merck, Marengo, Janssen, Related Sciences, Synthekine and Surface Oncology; is a founder of Danger Bio, Surface Oncology and Arsenal Biosciences; and is an inventor on a patent (US patent no. US10370446B2) submitted by Emory University that covers the use of PD-1 blockade to treat infections and cancer. A.S. is a consultant for Gritstone, Flow Pharma, CellCarta, Arcturus Therapeutics, Oxford Immunotec and Avalia Immunotherapies. La Jolla Institute for Immunology has filed for patent protection for various aspects of $\mathrm{T}$ cell epitope and vaccine design work. E.T.L.P. is consulting or is an advisor for Roche Diagnostics, ENPICOM, The Antibody Society, Immune Epitope Database and the American Autoimmune Related Diseases Association. D.J. is on the Biogen, Genentech, Novartis, EMD Serono, Banner Life Sciences, Bristol Myers Squibb and Sanofi-Genzyme advisory boards and has received research support (clinical trial site principal investigator) from Biogen, Genentech and University of California, Los Angeles. A.B.O. has participated as a speaker in meetings sponsored by and received consulting fees and/or grant support from Accure Therapeutics, Atara Biotherapeutics, Biogen, Bristol Myers Squibb/Celgene/Receptos, GlaxoSmithKline, Gossamer Bio, Janssen/Actelion, MedImmune, Merck/EMD Serono, Novartis, Roche/Genentech and Sanofi-Genzyme. All other authors declare no competing interests.

\section{Additional information}

Extended data is available for this paper at https://doi.org/10.1038/s41591-021-01507-2. Supplementary information The online version contains supplementary material available at https://doi.org/10.1038/s41591-021-01507-2.

Correspondence and requests for materials should be addressed to E. John Wherry, Rui Li or Amit Bar-Or.

Peer review information Nature Medicine thanks Howard Weiner, Lewis Au and the other, anonymous, reviewer(s) for their contribution to the peer review of this work. Saheli Sadanand was the primary editor on this article and managed its editorial process and peer review in collaboration with the rest of the editorial team.

Reprints and permissions information is available at www.nature.com/reprints. 


\begin{tabular}{|c|c|c|c|}
\hline & MS (n=20) & $H C(n=10)$ & $p$ - value \\
\hline Age, mean years \pm SD (range) & $40.35 \pm 8.44[27-57]$ & $35.2 \pm 9.8[25-61]$ & $p=0.146$ \\
\hline Females, n (\%) & $15(75)$ & $6(60)$ & $p=0.431$ \\
\hline Relapsing Remitting MS, n (\%) & $21(100)$ & - & \\
\hline \multicolumn{4}{|l|}{ Vaccine type } \\
\hline Pfizer, n (\%) & $12(61.9)$ & $8(80)$ & $p=0.419$ \\
\hline Moderna, n (\%) & $8(38.1)$ & $2(20)$ & \\
\hline $\begin{array}{l}\text { Time from last aCD20 to first vaccine } \\
\text { (weeks), mean } \pm S D \text { [range] }\end{array}$ & $19.77 \pm 9.52[2.6-41.1]$ & - & \\
\hline Prior cycles of aCD20, mean \pm SD [range] & $3.2 \pm 1.6[1-7]$ & - & \\
\hline \multicolumn{4}{|l|}{ Type of aCD20 } \\
\hline Ocrelizumab, n (\%) & $19(95)$ & - & \\
\hline Rituximab, n (\%) & $1(5)$ & - & \\
\hline EDSS, mean \pm SD [range] & $1.5 \pm 2.2(0-6.5)$ & - & \\
\hline
\end{tabular}

Extended Data Fig. 1 | Clinical characteristics of patients with MS treated with aCD20 and healthy controls. Clinical characteristics and demographics of the $\mathrm{HC}$ and MS-aCD2O cohorts. 


\begin{tabular}{l|ccc} 
& HC & MS- $\mathbf{C C D} 20$ & $\boldsymbol{p}$ - value \\
\hline anti-Spike IgG & & & \\
T2 & $100 \%$ & $28.57 \%$ & 0.0006 \\
T3 & $100 \%$ & $29.41 \%$ & 0.0004 \\
T4 & $100 \%$ & $82.35 \%$ & 0.2735 \\
T5 & $100 \%$ & $88.89 \%$ & 0.5238 \\
& & & \\
RBD Protein IgG & & & \\
T2 & $60 \%$ & $7.14 \%$ & 0.0088 \\
T3 & $100 \%$ & $17.65 \%$ & $<0.0001$ \\
T4 & $100 \%$ & $41.48 \%$ & 0.0031 \\
T5 & $100 \%$ & $50 \%$ & 0.0098 \\
& & & \\
\hline
\end{tabular}

Extended Data Fig. 2 | Spike and RBD serology positivity rates for patients with MS treated with aCD20 and healthy controls after SARS-CoV-2 mRNA vaccination. Anti-spike and anti-RBD IgG serological positivity rates in HC $(n=10)$ and MS-aCD20 patients $(n=20)$ across different time points. Two-sided Fisher's exact test was calculated for each timepoint. 
A

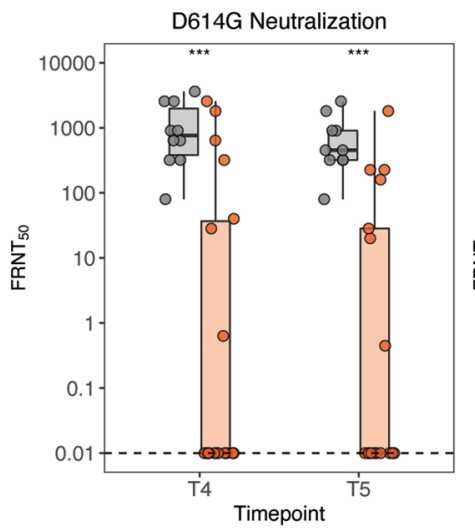

C

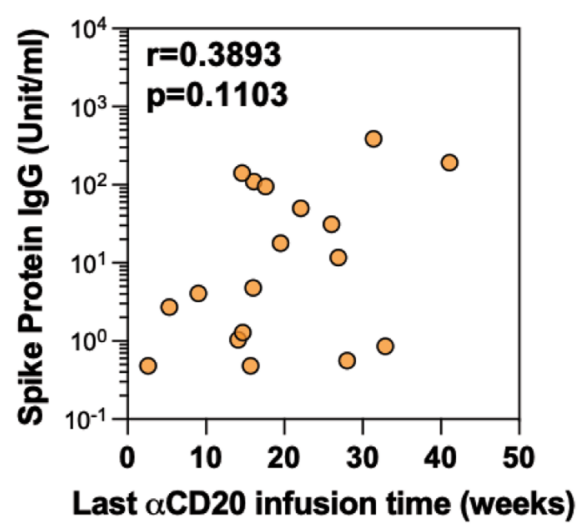

E

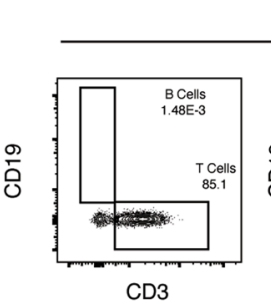

MS-aCD20

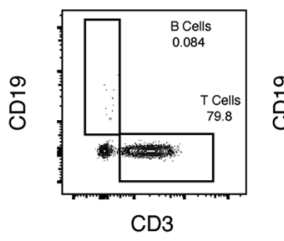

$\mathbf{F}$

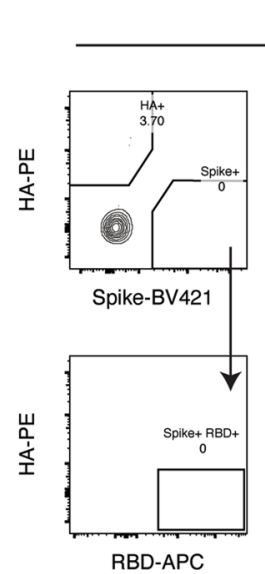

MS-aCD20

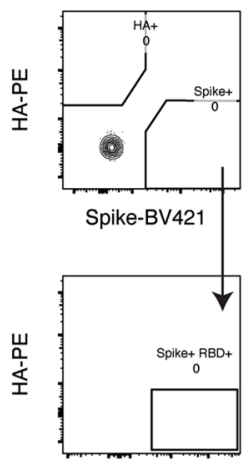

RBD-APC
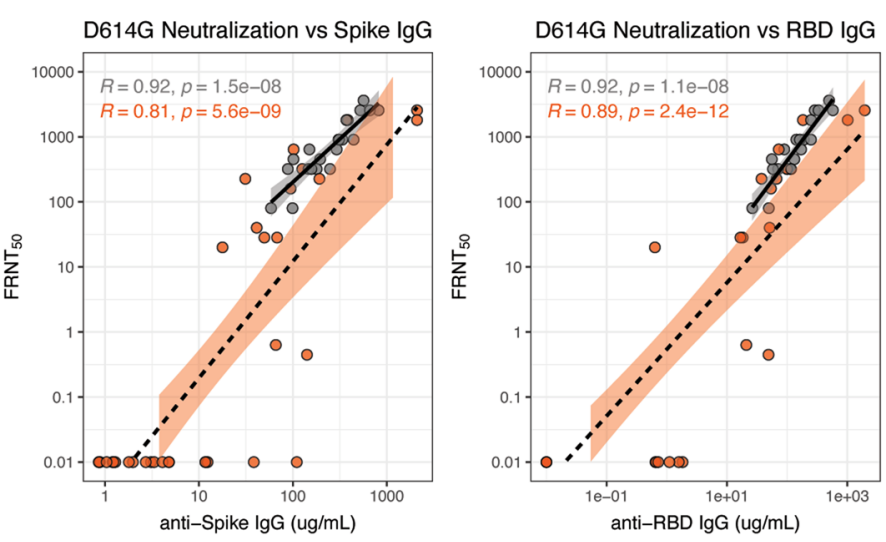

D

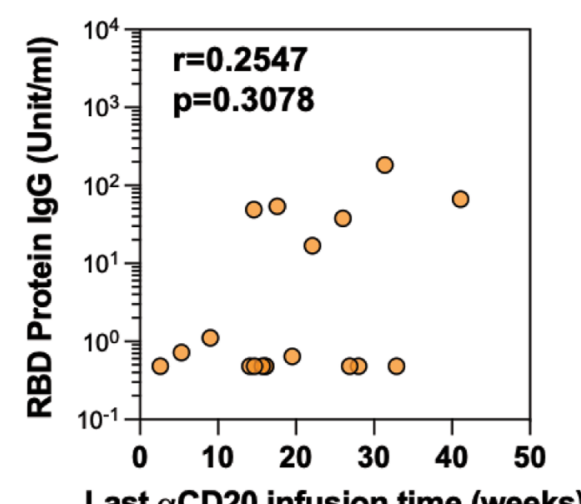

Last $\alpha$ CD20 infusion time (weeks)

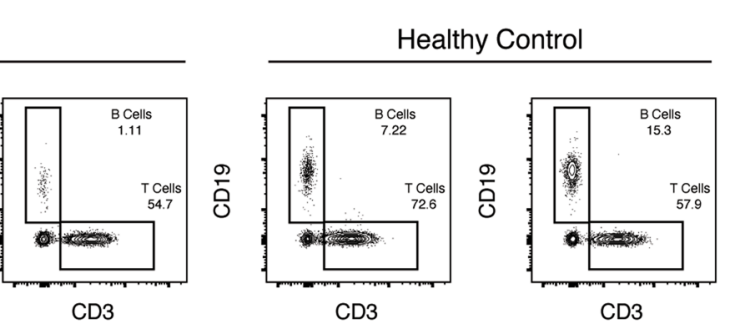


Extended Data Fig. 3 | Neutralization data, correlation of serologies with last aCD20 infusion, and B cell responses of patients with MS treated with aCD20 and healthy controls after SARS-CoV-2 mRNA vaccination. a) Tukey boxplots (median, Q1 and Q3 quartiles) of FRNT 50 values assayed against pseudotyped virus expressing SARS-CoV-2 D614G spike protein using serum samples from HCs ( $g r e y, n=10$ ) and MS-aCD20 patients (orange, $n=16$ ) at timepoints T4 and T5 that were positive for anti-spike IgG; unpaired Wilcoxon test $P$ values are shown. b) Spearman correlation analysis of anti-spike (left) and anti-RBD (right) IgG against D614G neutralization titers (HCs: grey, $n=10 ;$ MS-aCD20 patients, orange, $n=16$ ). c-d) Spearman correlation analysis between the weeks elapsed since last aCD20 infusion administration and anti-spike lgG (c) or anti-RBD IgG (d) at T5 for MS-aCD20 patients $(n=20)$. e) Gating strategy and representative plots for flow cytometric analysis of total B cells. $f)$ Gating strategy and representative plots for flow cytometric analysis of SARS-CoV-2-specific memory B cells. Cells were stained with fluorescently labeled SARS-CoV-2 full-length spike protein, SARSCoV-2 spike receptor binding domain (RBD), and influenza hemagglutinin (HA). Spike+ $\mathrm{HA}^{-}{ }^{-}$cells were subsequently analyzed for binding to RBD. 
A

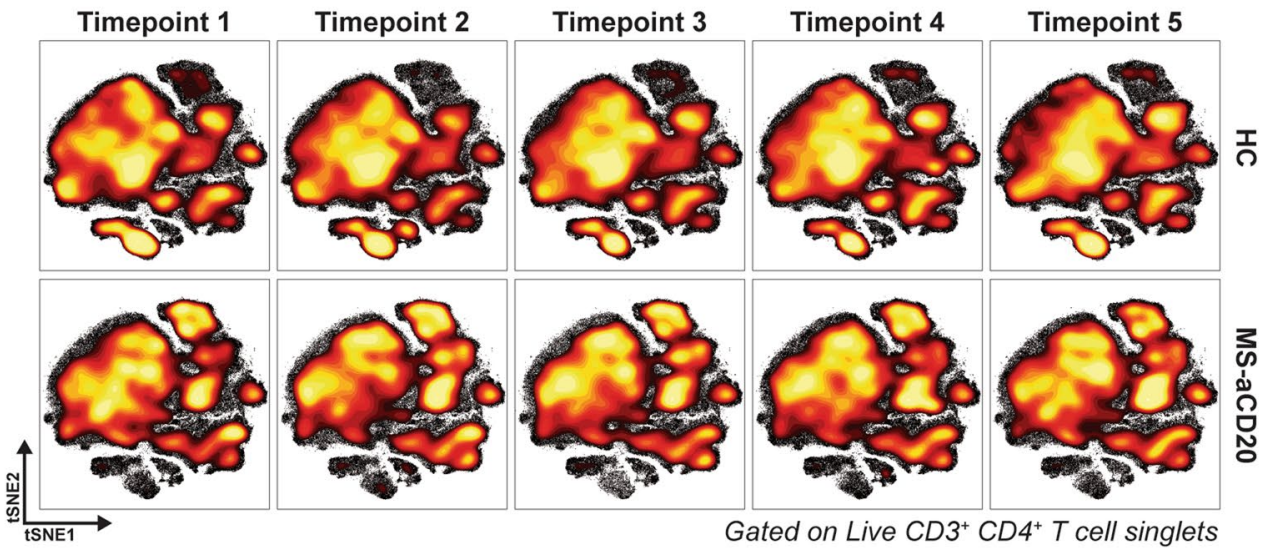

B

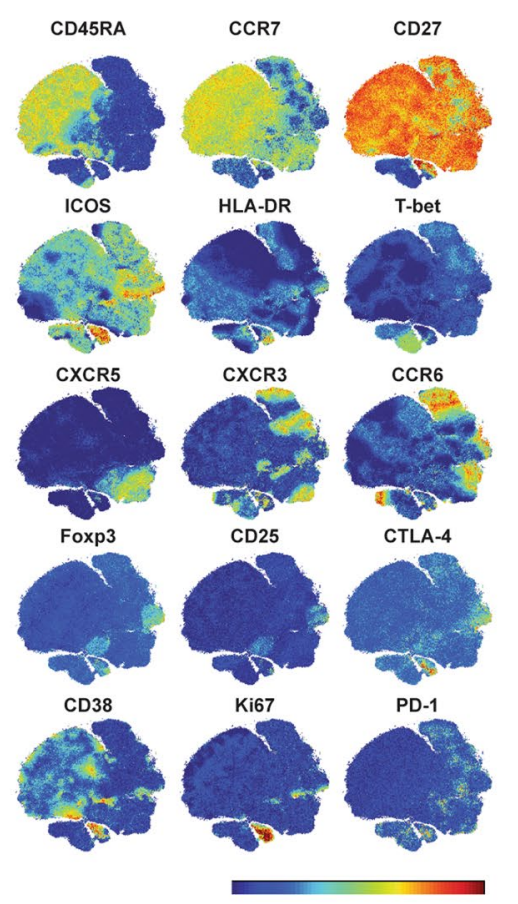

C
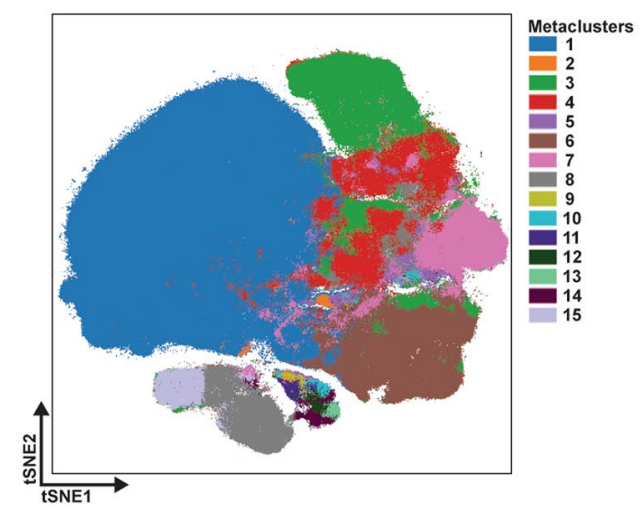

D

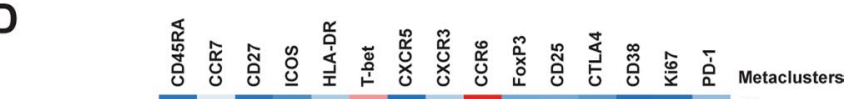

$\mathbf{E}$

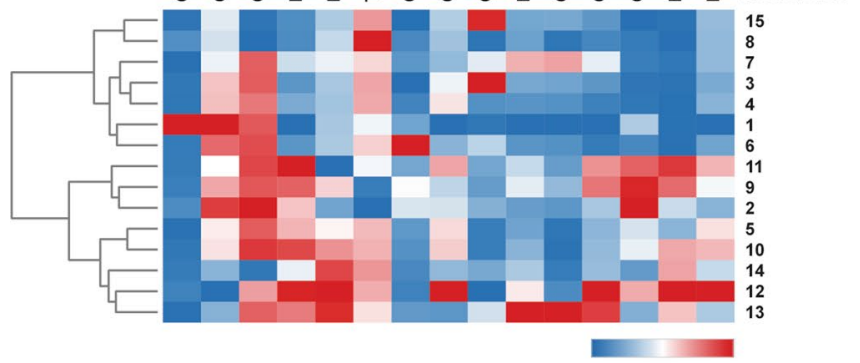

Timepoint 2

Timepoint 4
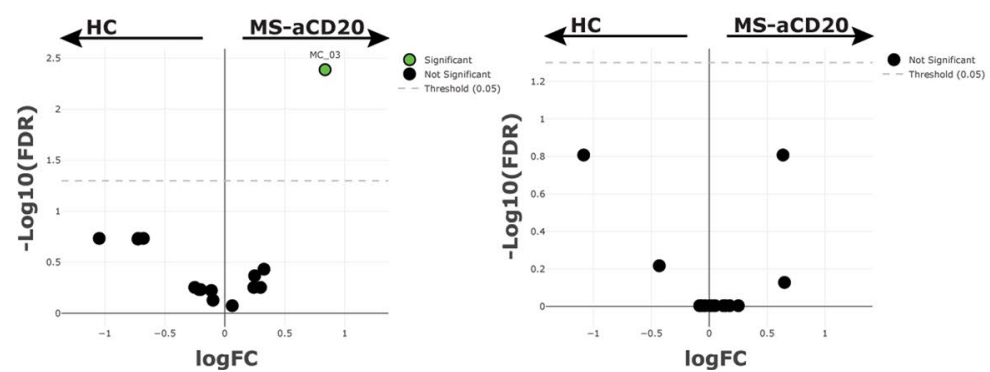

Extended Data Fig. 4 | See next page for caption. 
Extended Data Fig. 4 | CD4 T cell responses of patients with MS treated with aCD20 and healthy controls after SARS-CoV-2 mRNA vaccination. a) Opt-SNE projections of concatenated cytometry data for total CD3 ${ }^{+} C D 4^{+} T$ cells for each timepoint and group combination are shown. $b$ ) Surface expression intensity of the indicated markers projected on the opt-SNE 2D-map generated with all samples in (a) (color scale: MFI expression of each individual marker in a log scale). c) FlowSOM metaclusters were created using total $C D 3^{+} \mathrm{CD} 4^{+} \mathrm{T}$ cells concatenated from all samples and projected to the opt-SNE map. d) Surface expression intensity heatmap of the markers indicated for each of the 15 FlowSOM metaclusters in (c) (color scale: rowadjusted z-score expression for each individual marker). e) Volcano plots of the logFC (log fold change) of the abundance between the HC and MS-aCD20 groups for each of the 15 metaclusters indicated in (c) and the - $\log 10$ value of the false discovery rate (FDR) for timepoints 2 and 4 . HCs ( $n=10$ ) and MSaCD20 patients $(n=20)$. 
A
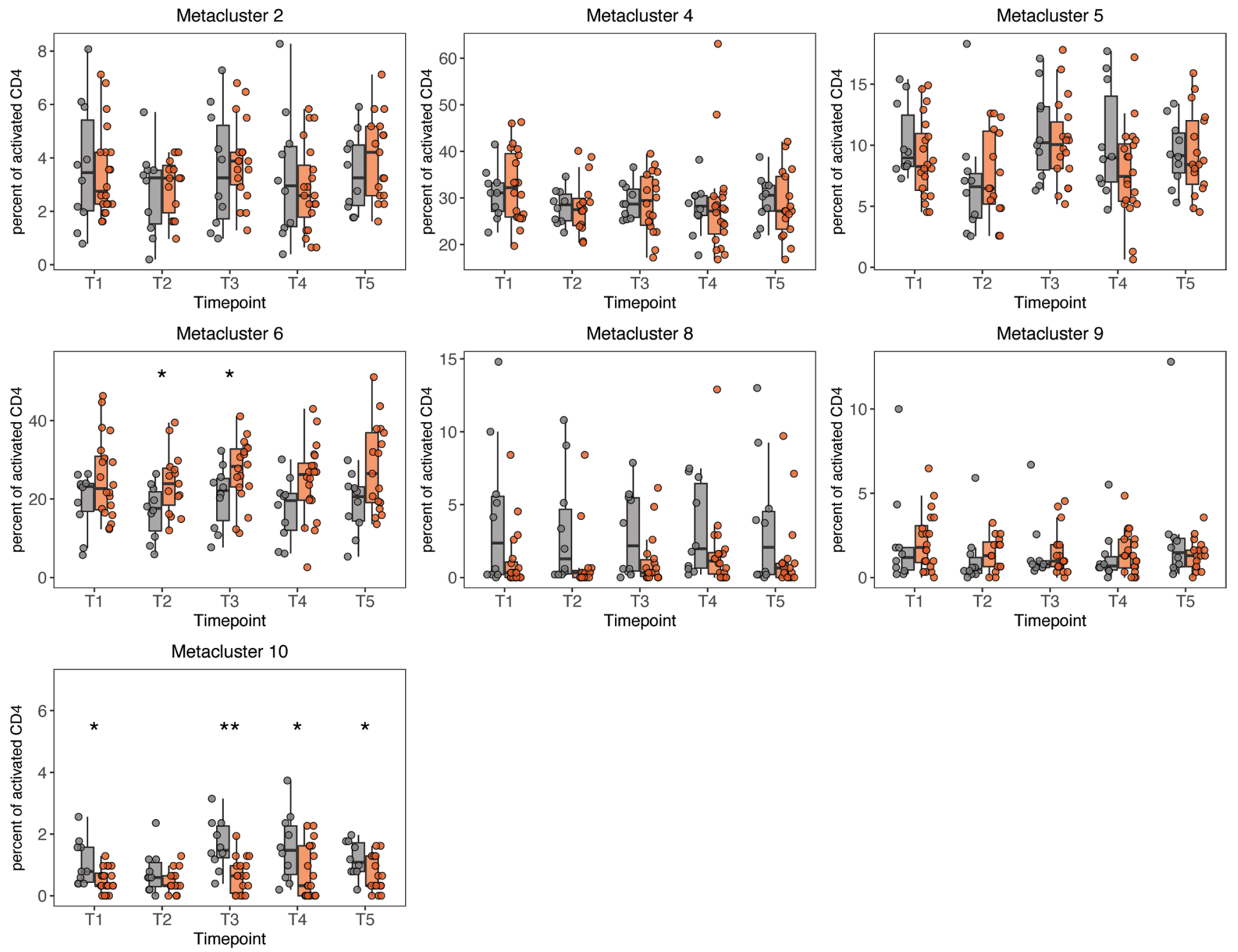

B

Metacluster 3

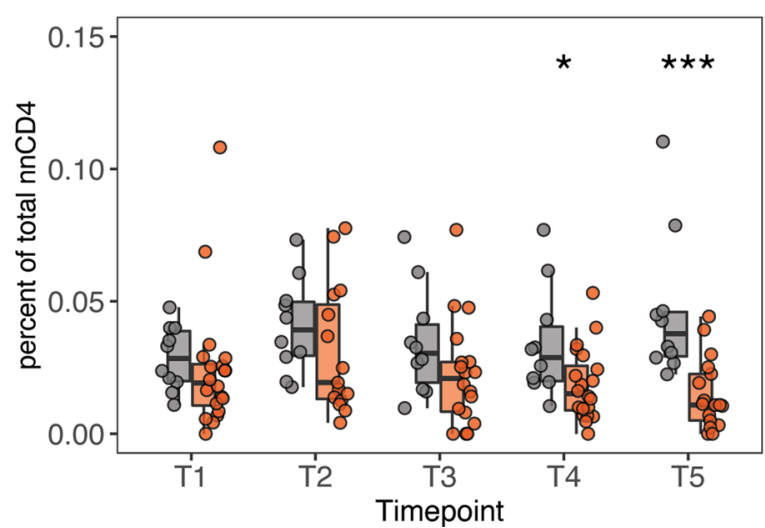

Extended Data Fig. 5 | FlowSOM metaclusters of activated CD4 T cells for patients with MS treated with aCD20 and healthy controls after SARS-CoV-2 mRNA vaccination. a) The abundance of metaclusters $2,4,5,6,8,9$ and 10 as percentage of activated Ki67 ${ }^{+} \mathrm{CD} 38^{+} \mathrm{CD} 4 \mathrm{~T}$ cells is shown for HC (grey) and MS-aCD20 (orange) groups; Tukey boxplots (median, Q1 and Q3 quartiles) are shown; unpaired, two-tailed Wilcoxon test $p$ values are depicted when $P<0.05$ between groups. b) The abundance of metacluster 3 as percentage of total non-naive CD4 T cells is shown for HC (grey) and MS-aCD20 (orange) groups; Tukey boxplots (median, Q1 and Q3 quartiles) are shown; unpaired, two-tailed Wilcoxon test $P$ values are depicted when $P<0.05$ between groups. HCs $(n=10)$ and MS-aCD20 patients $(n=20)$. For $P$ values: ${ }^{\star}$ indicates $P<0.05,{ }^{\star \star}$ indicates $P<0.01$, ${ }^{\star \star \star}$ indicates $P<0.001$. 
A
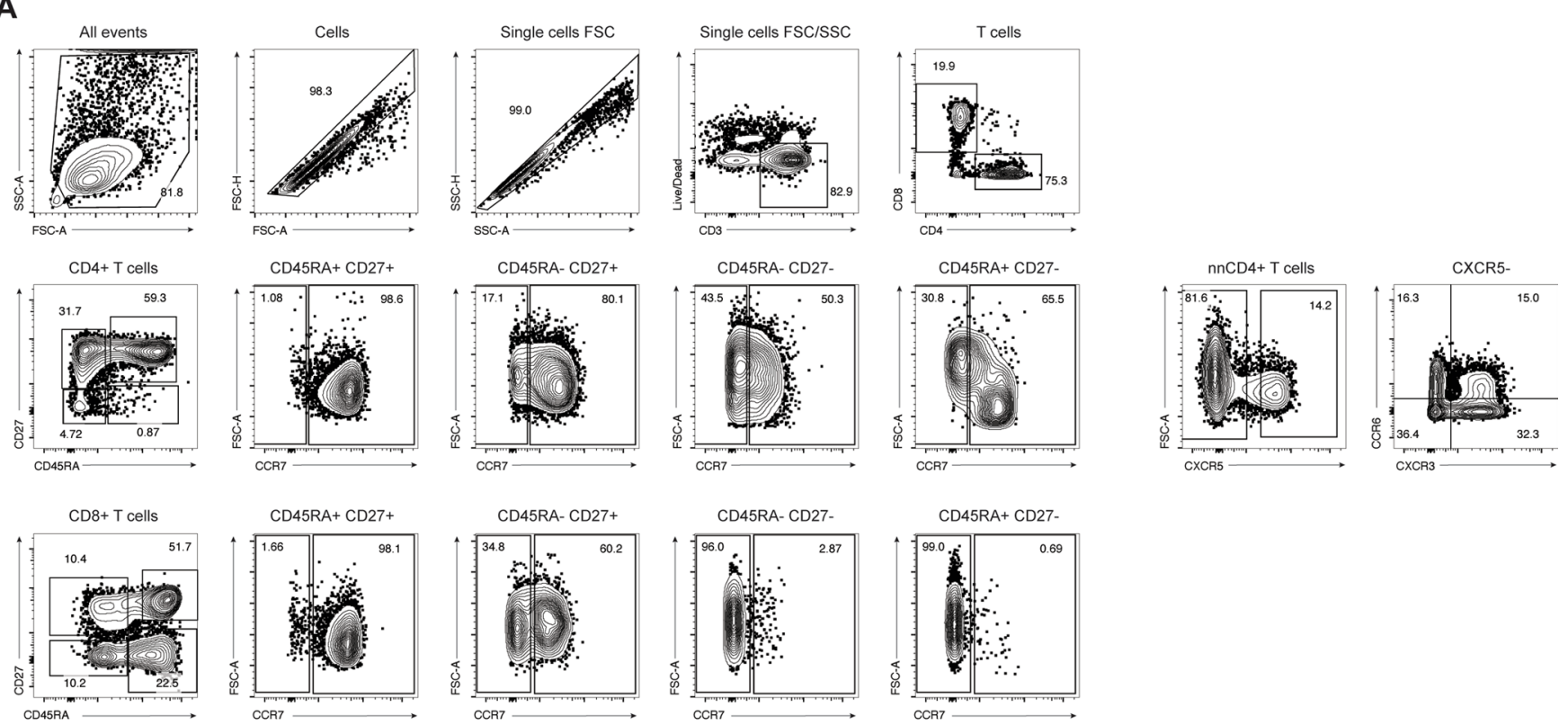

B
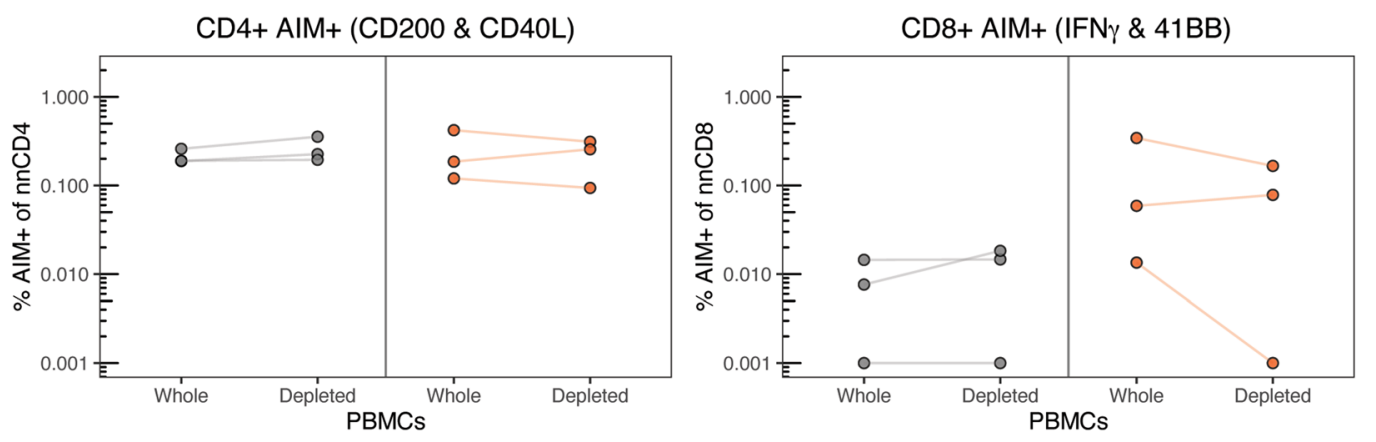

C
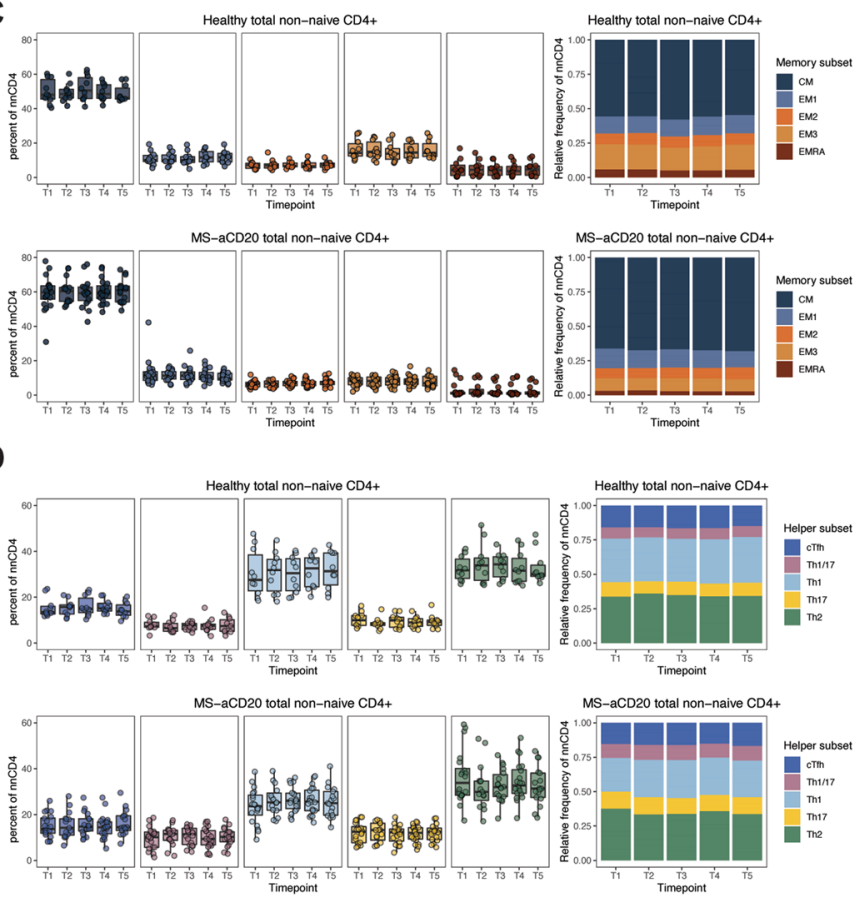

E
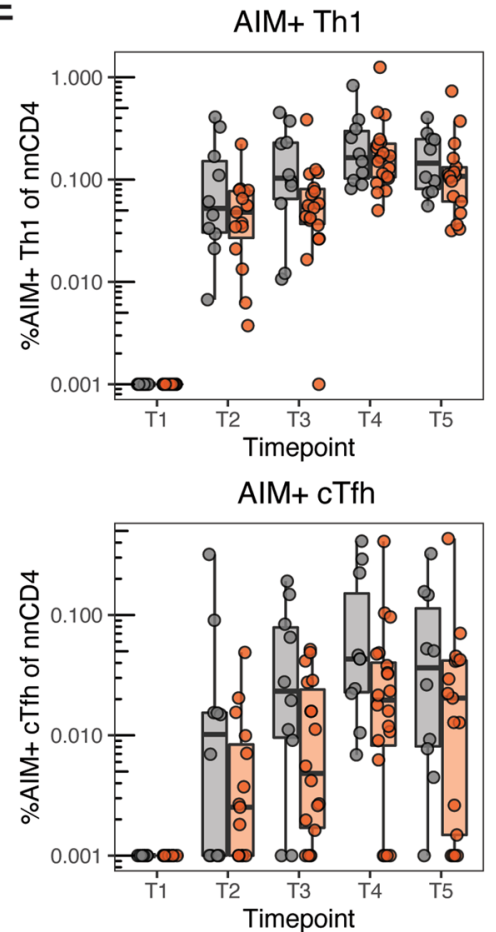

Extended Data Fig. 6 | See next page for caption. 
Extended Data Fig. 6 | AIM+ CD4 T cell gating strategy and total CD4 T cell memory and helper subsets in patients with MS treated with aCD20 and healthy controls after SARS-CoV-2 mRNA vaccination. a) Gating strategy for identifying T cell subsets. b) Timepoint 4 AIM+ CD4 T cell (left) and CD8 $T$ cell (right) frequency in whole PBMCs or PBMCs depleted of B cells by magnetic separation. Values represent the background-subtracted frequency of $\mathrm{AIM}^{+}$non-naive CD4 T cells above paired baseline frequencies. Lines connect paired samples from individual donors. Grey indicates $\mathrm{HC}(n=3)$; orange indicates MS-aCD20 patients $(n=3)$. c) Frequency of memory CD4 T cell subsets in total non-naive CD4 T cells. Left panels depict the percent of total non-naive $T$ cells that are in each subset. Right panels depict the relative frequency of each memory $T$ cell subset in the total non-naive population.

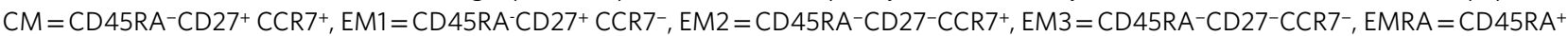
CD27-CCR7-. d) Frequency of T helper subsets in total non-naive CD4 T cells. Left panel depicts the percent of total non-naive $T$ cells that are helper $T$ cells in each subset. Right panel depicts the relative frequency of each helper $\mathrm{T}$ cell subset in the total non-naive population. $\mathrm{cTfh}=\mathrm{CXCR5^{+ } \text { , }}$

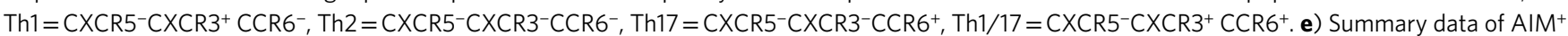
frequencies of the indicated $T$ cell populations following vaccination. Values represent the background-subtracted frequency of $A M^{+}$non-naive $T$ cells above paired baseline frequencies. Tukey boxplots (median, Q1 and Q3 quartiles) are shown; unpaired, two-tailed Wilcoxon test $P$ values are depicted when $P<0.05$ between the HC (grey, $n=10$ ) and MS-aCD20 (orange, $n=20$ ) groups. 

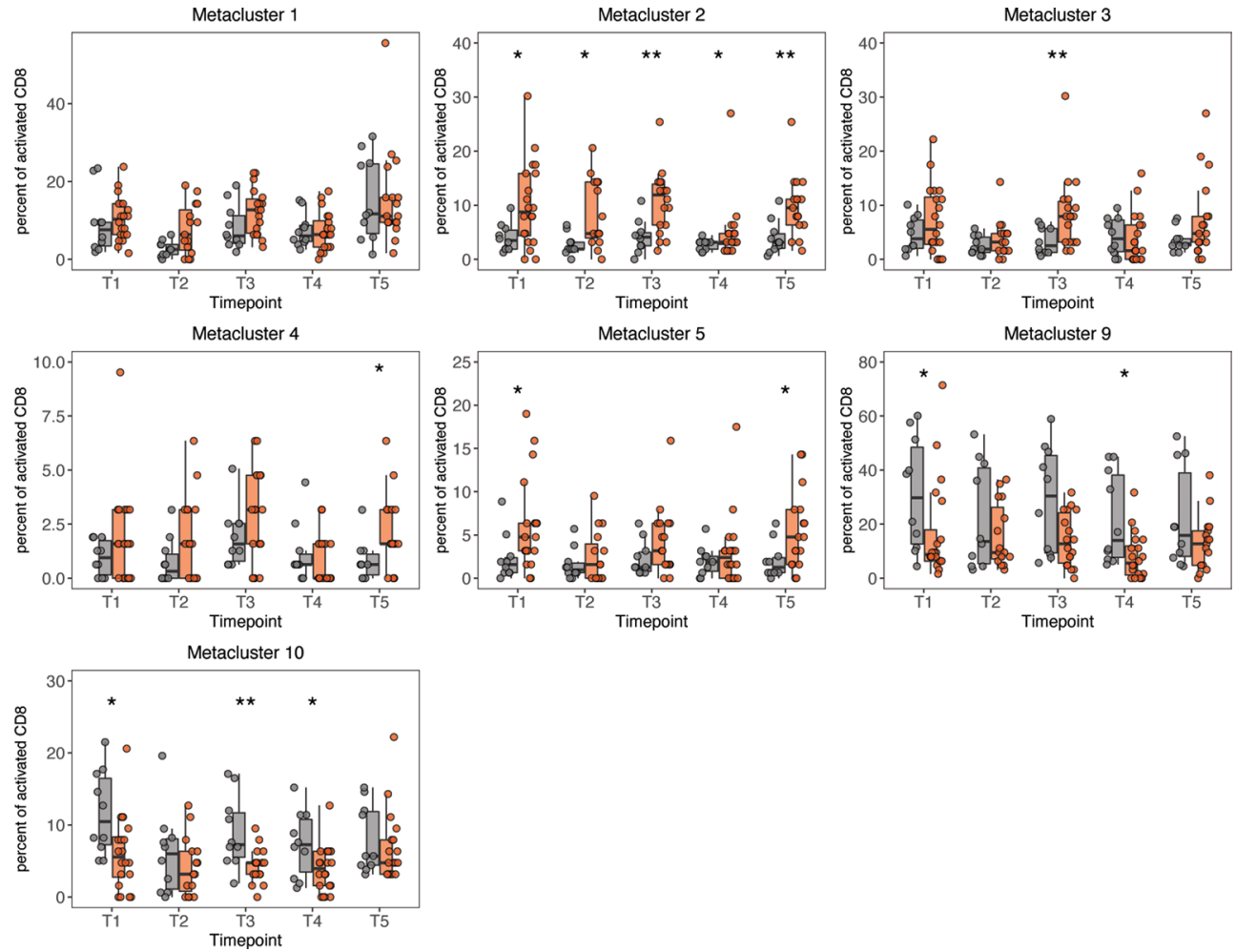

Extended Data Fig. 7 | FlowSOM metaclusters of activated CD8 T cells for patients with MS treated with aCD20 and healthy controls after SARS-CoV-2 mRNA vaccination. The abundance of metaclusters 1, 2, 3, 4, 5, 9 and 10 as percentage of activated Ki67+ CD38+CD8 T cells is shown for HC (grey, $\mathrm{n}=10$ ) and MS-aCD20 (orange, $\mathrm{n}=20$ ) groups; unpaired, two-tailed Wilcoxon test $\mathrm{p}$ values are shown when $\mathrm{P}<0.05$ between groups. For $P$ values: * indicates $P<0.05,{ }^{\star *}$ indicates $P<0.01$. 

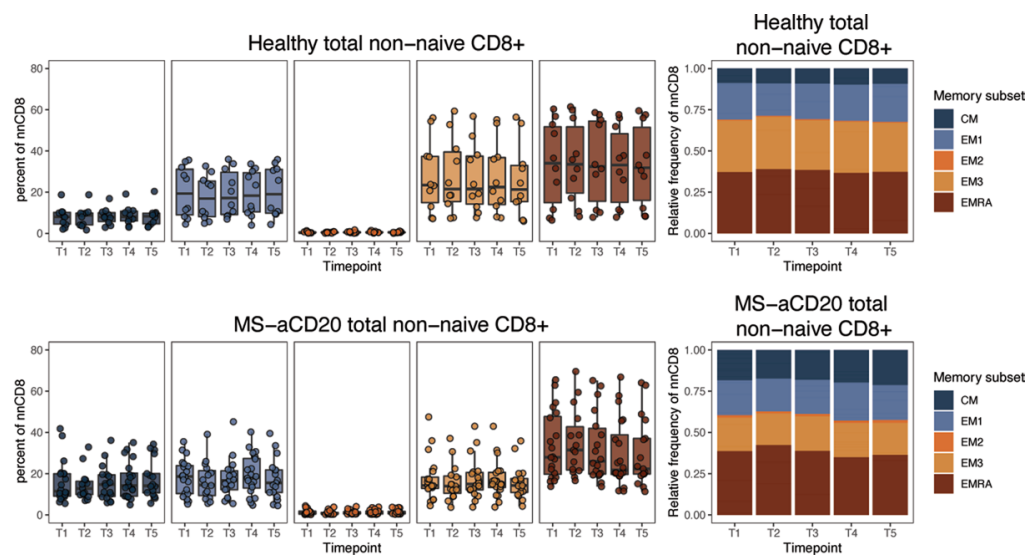

Extended Data Fig. 8. | Memory CD8 T cell subsets of patients with MS treated with aCD20 and healthy controls after SARS-CoV-2 mRNA vaccination. Frequency of memory CD8 T cell subsets in total non-naive CD8 T cells. Left panels depict the percent of total non-naive T cells that are in each subset. Right panels depict the relative frequency of each memory $T$ cell subset in the total non-naive population. $C M=C D 45 R A^{-} C D 27^{+} C C R 7^{+}$,

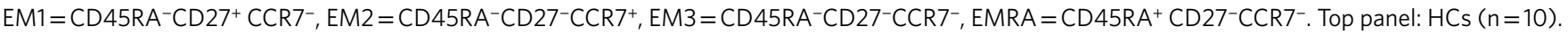
Bottom panel: $M S-a C D 20(n=20)$. 
A
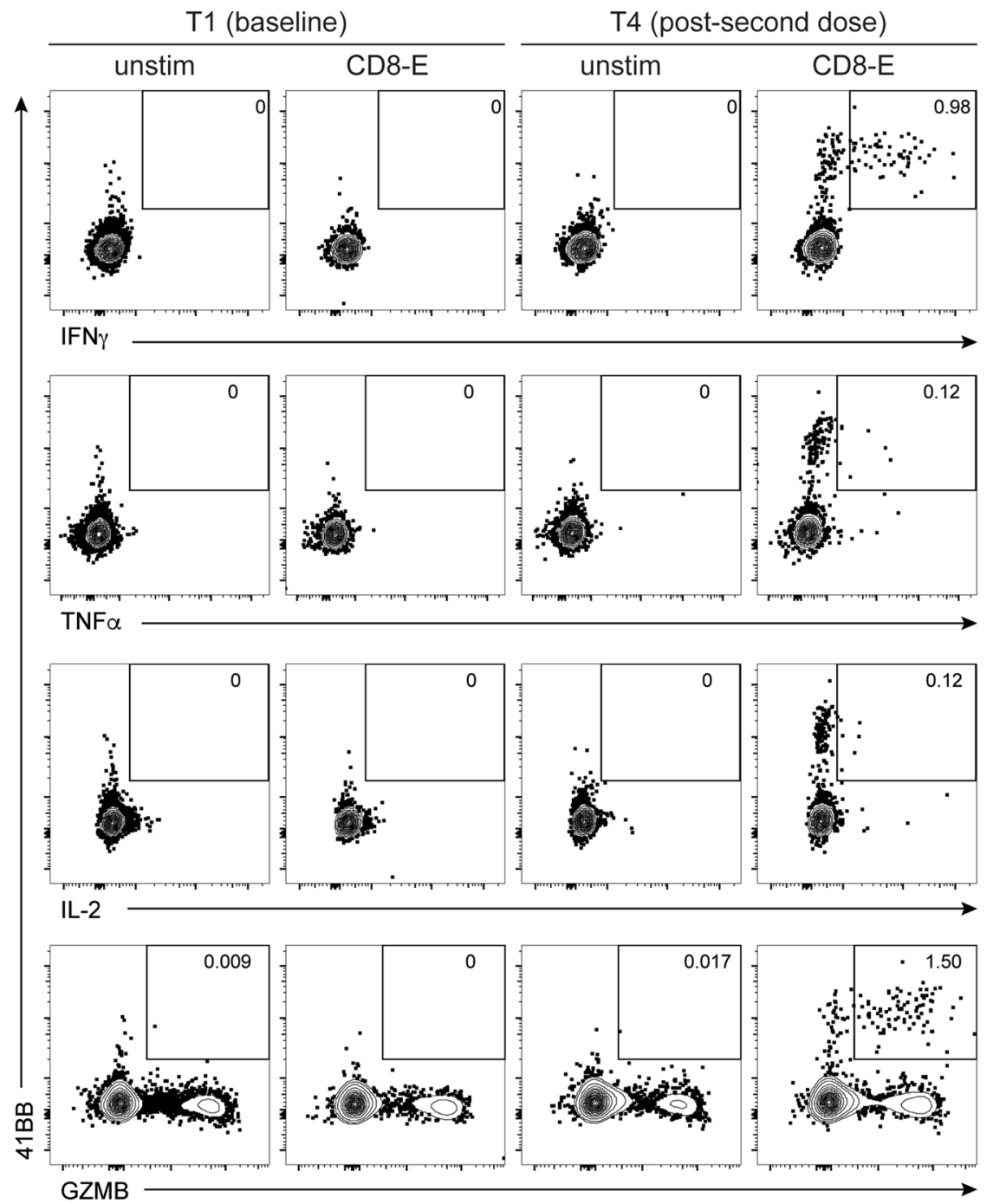

C

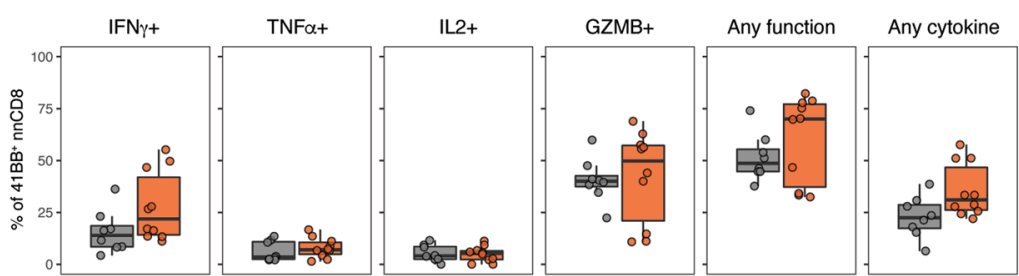

T4 (post-second dose)
B

Non-naive CD8 T cells
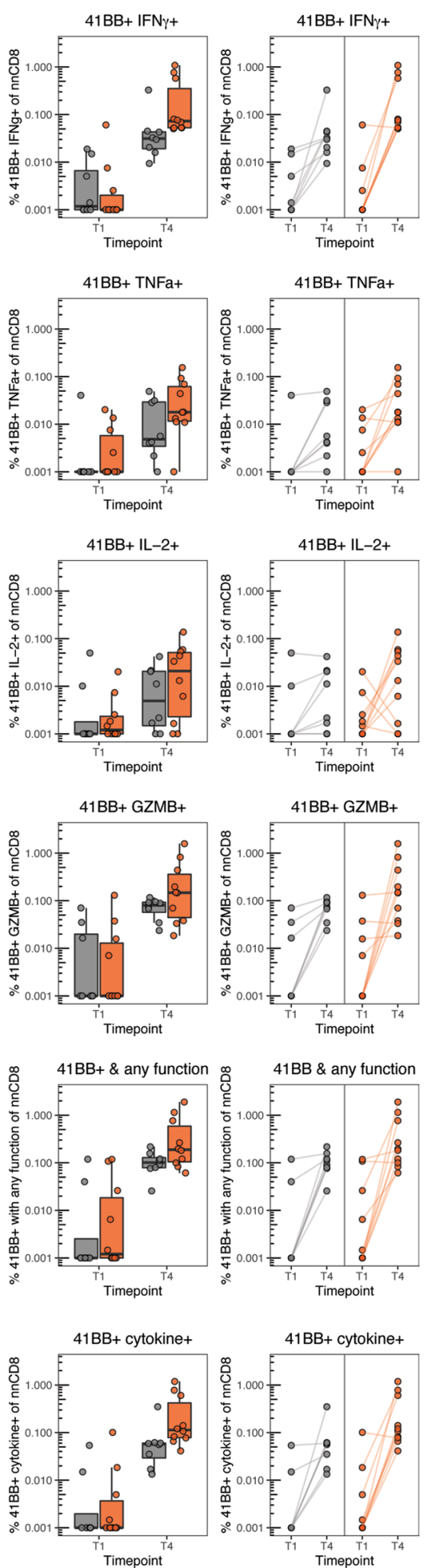

Extended Data Fig. 9 | Cytokine and Granzyme B production of AIM+ CD8 T cells of patients with MS treated with aCD20 and healthy controls after SARS-CoV-2 mRNA vaccination. a) Representative flow cytometry plots for quantifying IFN- $\gamma, \mathrm{TNF}$, IL-2 and Granzyme B expression in AIM+CD8 T cells. Numbers represent the percentage of $\mathrm{AIM}^{+}$non-naive CD8 T cells that co-express 41BB and the corresponding cytokine or Granzyme B. b) Tukey boxplots (median, Q1 and Q3 quartiles, left) and individual points (right) of the frequency of non-naive CD8 T cells that are 41BB ${ }^{+}$and express the indicated cytokines and/or Granzyme B at timepoints T1 and T4 following vaccination. Lines connect individual donors sampled longitudinally. Grey indicates HCs $(n=8)$, orange indicates MS-aCD20 patients $(n=10)$. c) Tukey boxplots (median, Q1 and Q3 quartiles) of the percentage of 41BB ${ }^{+}$non-naive CD8 T cells that express the indicated cytokines and/or Granzyme B at timepoint T4 following vaccination. Grey indicates HCs $(n=8)$, orange indicates MS-aCD20 patients $(n=10)$. Any function $=$ at least one of IFN- $\gamma, T N F, I L-2$, and Granzyme B. Any cytokine $=$ at least one of IFN- $\gamma$, TNF, and IL-2. 
A
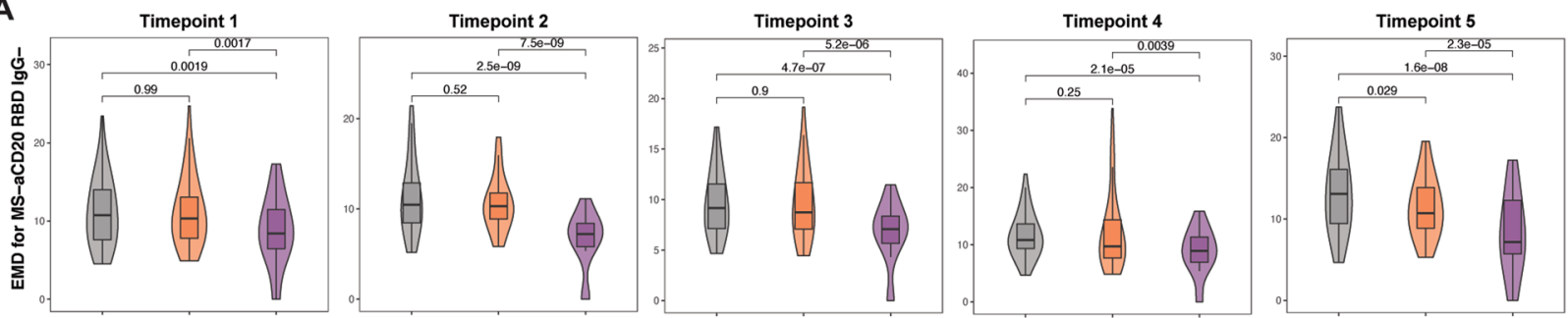

Subcohorts

Healthy
RBD Ab+

$\square$ MS-aCD20

MS-aCD20

B
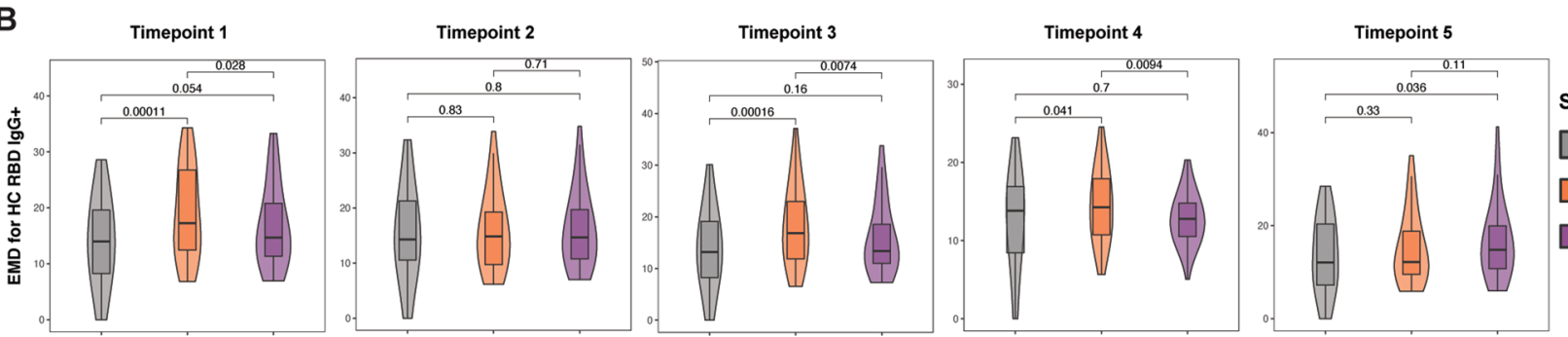

Subcohorts

Healthy

MS-aCD20

$\mathrm{RBD} A \mathrm{Ab}+$

MS-aCD20

RBD Ab-

Extended Data Fig. 10 | EMD analysis of activated CD4 and CD8 T cells of MS-aCD20 RBD antibody ${ }^{+}$and RBD antibody patients and healthy controls after SARS-CoV-2 mRNA vaccination. a) Violin plots representing the summary statistics (median, distribution) of the EMD distances to MS-aCD20 RBD Ab- samples on the activated CD4 T cell opt-SNE maps across all timepoints T1-T5 for the three groups: Healthy RBD Ab+, MS-aCD20 RBD Ab+ and MS-aCD20 RBD Ab-. Pairwise comparisons of means were done with unpaired, two-tailed Wilcoxon test and $P$ values are shown. $\mathbf{b}$ ) Violin plots representing the summary statistics (median, distribution) of the EMD distances to Healthy RBD Ab+ samples on the activated CD8 T cell opt-SNE maps across all timepoints T1-T5 for the three groups: Healthy RBD Ab+, MS-aCD20 RBD Ab+ and MS-aCD20 RBD Ab-. Pairwise comparisons of means were done with unpaired, two-tailed Wilcoxon test and $P$ values are shown. For each timepoint: Healthy RBD Ab+ (grey, $n=10$ ), MS-aCD20 RBD Ab+ (orange, $\mathrm{n}=10$ ) and MS-aCD20 RBD Ab- (purple, $\mathrm{n}=10$ ). 


\section{Reporting Summary}

Nature Portfolio wishes to improve the reproducibility of the work that we publish. This form provides structure for consistency and transparency in reporting. For further information on Nature Portfolio policies, see our Editorial Policies and the Editorial Policy Checklist.

\section{Statistics}

For all statistical analyses, confirm that the following items are present in the figure legend, table legend, main text, or Methods section.

$\mathrm{n} / \mathrm{a}$ Confirmed

$\bigotimes$ The exact sample size $(n)$ for each experimental group/condition, given as a discrete number and unit of measurement

\ A statement on whether measurements were taken from distinct samples or whether the same sample was measured repeatedly

The statistical test(s) used AND whether they are one- or two-sided

Only common tests should be described solely by name; describe more complex techniques in the Methods section.

\ A description of all covariates tested

$\square$ A description of any assumptions or corrections, such as tests of normality and adjustment for multiple comparisons

$\checkmark$ A full description of the statistical parameters including central tendency (e.g. means) or other basic estimates (e.g. regression coefficient)

AND variation (e.g. standard deviation) or associated estimates of uncertainty (e.g. confidence intervals)

For null hypothesis testing, the test statistic (e.g. $F, t, r$ ) with confidence intervals, effect sizes, degrees of freedom and $P$ value noted Give $P$ values as exact values whenever suitable.

Х $\square$ For Bayesian analysis, information on the choice of priors and Markov chain Monte Carlo settings

Х $\square$ For hierarchical and complex designs, identification of the appropriate level for tests and full reporting of outcomes

$\square \bigotimes$ Estimates of effect sizes (e.g. Cohen's $d$, Pearson's $r$ ), indicating how they were calculated

\section{Our web collection on statistics for biologists contains articles on many of the points above.}

\section{Software and code}

Policy information about availability of computer code

Data collection All FACS-based data collection was done with BD FACS Diva Software (2019 release)

Data analysis Data Analysis was done with the following software: R (Version 4.0.5), R Studio (Version 1.4.1106), emdist package (version 0.3-1), OMIQ (https://app.omiq.ai/, release 2021) and GraphPad Prism (Version 9.1.2)

For manuscripts utilizing custom algorithms or software that are central to the research but not yet described in published literature, software must be made available to editors and

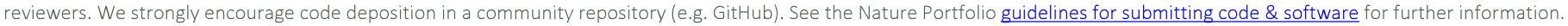

\section{Data}

Policy information about availability of data

All manuscripts must include a data availability statement. This statement should provide the following information, where applicable:

- Accession codes, unique identifiers, or web links for publicly available datasets

- A description of any restrictions on data availability

- For clinical datasets or third party data, please ensure that the statement adheres to our policy

\footnotetext{
All data are available in the main Text, Figures, Extended Data Figures and supplementary materials. Raw .fcs files can be accessed through the following links: - flow cytometry files for B cell analysis (Figure 1, Extended Data Figure 3):

https://premium.cytobank.org/cytobank/experiments/378970

- flow cytometry files for high-dimensional analysis (Figures 2, 4, 6 and Extended Data Figures 4, 5, 7, 10 and Supplementary Figure 1):

https://premium.cytobank.org/cytobank/experiments/378712

- flow cytometry files for AIM T cell analysis (Figures 3, 5, 6 and Extended Data Figures 6, 8)
} 


\title{
Field-specific reporting
}

Please select the one below that is the best fit for your research. If you are not sure, read the appropriate sections before making your selection.

$\bigotimes$ Life sciences

Behavioural \& social sciences

Ecological, evolutionary \& environmental sciences

For a reference copy of the document with all sections, see nature.com/documents/nr-reporting-summary-flat.pdf

\section{Life sciences study design}

All studies must disclose on these points even when the disclosure is negative.

Sample size

Based on our previous studies (Goel et al, Sci Immunology, 2021) that utilized 40 healthy individuals receiving SARS-CoV-2 vaccination and based on the large effect sizes we noted at the post-vaccine timepoints (plus the published serological differences of MS patients compared to healthy volunteers following vaccination), we estimated that a total longitudinal cohort of 30 participants (20 MS and 10 healthy) would provide sufficient $\mathrm{n}$ to detect differences. The primary statistical goal was to compare across groups, and an F-test power calculation for three groups of 10 subjects each (which was the analysis with the fewest number of subjects per group, with three groups: HC, MS RBD IgG-, MS RBD $\operatorname{lgG}+$ ), assuming conservatively a modest effect size of $0.6-0.7$, provides a power calculation of $>0.8$ at an a significance level of 0.05 .

Data exclusions We excluded one patient with mutliple sclerosis who clinically had COVID-19 in the past. All healthy participants were clinically COVID-19 naive. We thus wanted to match the two groups and assess cleanly the immune response of a naive immune system to SARS-CoV-2 mRNA vaccination.

Replication No experimental replication was conducted in this manuscript, as all samples were derived from primary human participants with a predefined longitudinal cohort study design.

Randomization The study design did not allow for randomization (the two groups included MS patients vs HCs; all participants received mRNA vaccination). All subjects were enrolled sequentially as they agreed to participate. Covariates like age and sex were controlled by assessing these variables in both groups periodically during recruitment.

Blinding

Blinding was performed at the time of data collection. All researchers involved in data collection were blinded to the group allocation of the samples. All investigators were unblinded during the analysis to allow comparisons among the different groups.

\section{Reporting for specific materials, systems and methods}

We require information from authors about some types of materials, experimental systems and methods used in many studies. Here, indicate whether each material, system or method listed is relevant to your study. If you are not sure if a list item applies to your research, read the appropriate section before selecting a response.

\begin{tabular}{l|l} 
Materials \& experimental systems \\
\hline $\mathrm{n} / \mathrm{a}$ & Involved in the study \\
\hline & $\bigotimes$ Antibodies \\
$\square$ & $\square$ Eukaryotic cell lines \\
$\square$ & $\square$ Animals and other organisms \\
$\square$ & $\square$ Clinical data \\
$\square$ & $\square$ Dual use research of concern
\end{tabular}

\begin{tabular}{l|l} 
Methods \\
\hline n/a & Involved in the study \\
$\square$ & $\square$ ChIP-seq \\
$\square$ & $\bigotimes$ Flow cytometry \\
$\searrow$ & $\square$ MRI-based neuroimaging
\end{tabular}

\section{Antibodies}

Antibodies used

\author{
High-dimensional FACS: \\ CD27 BUV 395 Clone L128 BD Cat\# 563815 Dilution 1:200 \\ CD71 BUV 496 Clone M-A712 BD Cat\# 750652 Dilution 1:50 \\ CD3 BUV 563 Clone UCHT1 BD Cat\# 748569 Dilution 1:200 \\ CD8 BUV 615 Clone RPA-T8 BD Cat\# 751518 Dilution 1:1600 \\ CD38 BUV 661 Clone HIT2 BD Cat\# 612969 Dilution 1:200 \\ CCR6 BUV 737 Clone 11A9 BD Cat\# 612780 Dilution 1:100 \\ HLA-DR BUV 805 Clone G46-6 BD Cat\# 748338 Dilution 1:200 \\ CTLA4 BV 421 Clone BNI3 BD Cat\# 562743 Dilution 1:100
}


PD-1 BV 480 Clone EH12.11 BD Cat\# 566112 Dilution 1:50

CCR7 BV 510 Clone G043H7 Biolegend Cat\# 353232 Dilution 1:100

Zombie Yellow BV 570 - Biolegend Cat\# 423103 Dilution 1:500

CD45RA BV 605 Clone HI100 Biolegend Cat\# 304134 Dilution 1:100

CD25 BV 650 Clone M-A251 BD Cat\# 563719 Dilution 1:100

CXCR3 BV 711 Clone G025H7 Biolegend Cat\# 353732 Dilution 1:100

CD4 BV 750 Clone SK3 BD Cat\# 566355 Dilution 1:2000

ICOS BV 785 Clone C398.4A Biolegend Cat\# 313534 Dilution 1:50

SLAM AF 488 Clone A12 (7D4) Biolegend Cat\# 306312 Dilution 1:50

CD127 BB 700 Clone HIL-7R-M21 BD Cat\# 566398 Dilution 1:100

CXCR4 PE-Cy5 Clone 12G5 Biolegend Cat\# 306508 Dilution 1:500

FoxP3 PE-Cy5.5 Clone PCH101 Fisher Cat\# 35-4776-42 Dilution 1:50

Ki67 PE-Cy7 Clone B56 BD Cat\# 561283 Dilution 1:400

T-bet AF 647 Clone 4B10 Biolegend Cat\# 644804 Dilution 1:200

CXCR5 APC-R700 Clone RF8B2 BD Cat\# 565191 Dilution 1:50

Bcl-6 APC-Cy7 Clone K112-91 BD Cat\# 563581 Dilution 1:100

AIM assays:

BUV395 CD4 BD Biosciences Clone SK3 Cat\#563550 Dilution 1:400

BUV496 CD8 BD Biosciences Clone RPA-T8 Cat\#612943 Dilution 1:400

BUV615 CD45RA BD Biosciences Clone HI100 Cat\#751555 Dilution 1:2000

BUV737 CD27 BD Biosciences Clone L128 Cat\#612829 Dilution 1:400

BUV805 CD3 BD Biosciences Clone UCHT1 Cat\#612896 Dilution 1:800

BV421 CXCR3 Biolegend Clone G02587 Cat\#353716 Dilution 1:800

BV650 CCR7 Biolegend Clone G043H7 Cat\#353234 Dilution 1:400

BV605 CD69 Biolegend Clone FN50 Cat\#310938 Dilution 1:400

BV711 CD40L Biolegend Clone 24-31 Cat\#310838 Dilution 1:50

BV785 CD107a Biolegend Clone H4A3 Cat\#328644 Dilution 1:100

FITC IFNy Biolegend Clone 4S.B3 Cat\#502515 Dilution 1:400

PE CD200 Biolegend Clone A18042B Cat\#399804 Dilution 1:100

PE-Cy7 OX40 Biolegend Clone Ber-ACT35 Cat\#350012 Dilution 1:1600

AF647 41BB Biolegend Clone 4B4-1 Cat\#309810 Dilution 1:400

APC-R700 CXCR5 BD Biosciences Clone RF8B2 Cat\#565191 Dilution 1:100

APC-Cy7 CCR6 Biolegend Clone G034E3 Cat\#353432 Dilution 1:800

BV421 IL-2 BD Biolegend Clone MQ1-17H12 Cat\# 500328 Dilution 1:500

PE-Texas Red Granzyme B ThermoFisher Clone GB11 Cat\#GRB17 Dilution 1:3200

PE TNF alpha ThermoFisher Clone MAb11 Cat\# 12-7349-82 Dilution 1:800

Miscellaneous:

Monoclonal antibody CR3022: plasmids to express CR3022 were provided by I. Wilson (Scripps)

Anti-VSV-G [1E9F9], clone 1E9F9, Absolute Antibody, cat\# Ab01402-2.0, concentration of $600 \mathrm{ng} / \mathrm{ml}$

Validation

All antibodies are validated by the manufacturer and are quality control tested by surface or intracellular immunofluorescent staining with flow cytometric analysis. For more information on the antibodies used, please visit biolegend.com, bdbiosciences.com and thermofisher.com.

Eukaryotic cell lines

Policy information about cell lines

Cell line source(s)

ATCC

Authentication

Quality control specifications as provided by the supplier (STR profile):

CSF1PO: 11,12

D13S317: 12,14

D16S539: 9,13

D5S818: 8,9

D7S820: 11

TH01: 7, 9.3

TPOX: 11

vWA: 16,19

Amelogenin: $X$

Mycoplasma contamination

All cell lines tested negative by the supplier for mycoplasma contamination

Commonly misidentified lines

(See $\underline{I C L A C}$ register)

Name any commonly misidentified cell lines used in the study and provide a rationale for their use.

Human research participants

Policy information about studies involving human research participants

Population characteristics

Among the 20 participants with multiple sclerosis, all were adults with an age range of 27-57 years (mean age of 40 ), with females comprising $75 \%$. All of them had a diagnosis of relapsing-remitting multiple sclerosis, and all were being treated with 
an anti-CD20 monoclonal antibodies as monotherapy during the period of the study. All 10 participants in the healthy control group were people with no known autoimmune conditions or on any immune-modulating therapies, fell within the age range of 25-61 years (mean age of 35 years), with $60 \%$ of them being females. None of the 30 total participants included in the manuscript had a clinical history of COVID-19.

Recruitment

Participants with multiple sclerosis were recruited through the Multiple Sclerosis clinic at University of Pennsylvania Health System (UPHS). Patients who were about to get vaccinated were either identified by their primary neurologist and referred to the study recruiting team, or were identified through the vaccination clinic organized by UPHS. They were then contacted via phone or in-person and explained about the study goals, risks/benefits and requirements. Healthy controls were recruited through a word-of-mouth and group emails among employees at the University of Pennsylvania. No compensation was provided. All the participants were recruited in the early weeks to months after SARS-CoV-2 vaccinations were approved. Vaccinations at the time were being offered to participants by the 'category' they belonged to, as deemed eligible through public health and institutional recommendations. Therefore, participants were recruited in the order that they were offered vaccinations, which is unlikely to have played into selection bias by investigators. One potential means for self-selection bias could be participants' willingness for serial venipuncture, but it is unlikely that this could have impacted any of the results.

Ethics oversight

Institutional Review Board, University of Pennsylvania

Note that full information on the approval of the study protocol must also be provided in the manuscript.

\section{Flow Cytometry}

Plots

Confirm that:

\The axis labels state the marker and fluorochrome used (e.g. CD4-FITC).

\The axis scales are clearly visible. Include numbers along axes only for bottom left plot of group (a 'group' is an analysis of identical markers).

\All plots are contour plots with outliers or pseudocolor plots.

\A numerical value for number of cells or percentage (with statistics) is provided.

\section{Methodology}

Sample preparation

Instrument

Software

Cell population abundance

Gating strategy
For all studies, PBMCs were thawed and promptly washed. Subsequently, we proceeded with either antibody staining directly or in vitro activation with peptide pools and then antibody-based staining.

Samples were acquired on a 5 laser BD FACS Symphony A5 (X50 SORP).

Events were acquired with BD FACS Diva Software (release 2019)

No sorting experiments were conducted. For FACS-based event analysis, standardized SPHERO rainbow beads (Spherotech, Cat\#RFP-30-5A) were used to track and adjust PMTs over time. UltraComp eBeads (ThermoFisher, Cat\#01-2222-42) were used for compensation. Up to $1 \times 10^{\wedge} 6$ PBMCs were acquired per each sample.

Gating strategies are shown in the Extended Data. Briefly, all lymphocytes were initially gated using standard FSC/SSC gating, followed up by singlet discrimination. All subsequent manual gating was done with markers optimized to have clear distinction between positive and negative populations.

$\bigotimes$ Tick this box to confirm that a figure exemplifying the gating strategy is provided in the Supplementary Information. 